\title{
Height pairings, exceptional zeros and Rubin's formula: the multiplicative group
}

\author{
Kâzim Büyükboduk
}

\begin{abstract}
In this paper we prove a formula, much in the spirit of one due to Rubin, which expresses the leading coefficients of various $p$-adic $L$-functions in the presence of an exceptional zero in terms of Nekováŕ's $p$-adic height pairings on his extended Selmer groups. In a particular case, the Rubin-style formula we prove recovers a $p$-adic Kronecker limit formula. In a disjoint case, we observe that our computations with Nekovár's heights agree with the Ferrero-Greenberg formula (more generally, Gross' conjectural formula) for the leading coefficient of the KubotaLeopoldt $p$-adic $L$-function (resp., the Deligne-Ribet $p$-adic $L$-function) at $s=0$.
\end{abstract}

Mathematics Subject Classification (2010). Primary 11R23, 11R34, 11R42; Secondary $11 \mathrm{G} 50$.

Keywords. Zeros, cyclotomic units, height pairings, $p$-adic $L$-functions.

\section{Contents}

1 Introduction . . . . . . . . . . . . . . . . . 71

2 Height pairings on extended Selmer groups . . . . . . . . . . . . 76

3 Cyclotomic units . . . . . . . . . . . . . . . . . . . 83

4 Computation of the height pairing . . . . . . . . . . . . . 87

5 Rubin's formula . . . . . . . . . . . . . . . . . . . 91

6 p-adic L-functions and Nekováŕr's height pairing . . . . . . . . . . . . . . 97

References . . . . . . . . . . . . . . . . . . . 108

\section{Introduction}

The celebrated formula of Gross and Zagier [GZ86] expresses the first derivative at $s=1$ of a Rankin $L$-series of a modular form $f$ of weight 2 on $\Gamma_{0}(N)$ in terms of the Néron-Tate height of a Heegner point on the $f$-quotient $A_{f}$ of the Jacobian $J_{0}(N)$ 
of the modular curve $X_{0}(N)$. A $p$-adic variant of this formula has been proved by Perrin-Riou [PR87], relating the $p$-adic height of a Heegner point on $A_{f}$ to a first derivative (taken in the cyclotomic direction) of a two-variable $p$-adic $L$-function associated to $f$. (See also [How05] for a generalization of this formula with more Iwasawa theoretical flavor). Later, Nekovár [Nek95] extended the results of [PR87] to higher weight modular forms, where he utilized his $p$-adic heights defined earlier in [Nek93].

When $E$ is an elliptic curve defined over $\mathbb{Q}$ with $\mathrm{CM}$ and $p$ is an odd prime at which $E$ has good, ordinary reduction, Perrin-Riou [PR83] gives a purely algebraic construction of the canonical $p$-adic height pairing on the $p$-adic Selmer group $S_{p}(E / \mathbb{Q})$. If further $L(E / \mathbb{Q}, 1)=0$, Rubin [Rub92] obtains a formula for the special values of the associated Katz two-variable $p$-adic $L$-function in terms of the $p$-adic height of an element $x_{p} \in S_{p}(E)$ (which is constructed from elliptic units). When $E$ does not have CM, but still good, ordinary at $p$, results along this line have been obtained by Perrin-Riou [PR93] utilizing Nekovář's definition of $p$-adic heights [Nek93] and Kato's zeta-elements [Kat04]. Perrin-Riou's formula in [PR93] goes hand-in-hand with Rubin's result [Rub94], Theorem 1 (which follows from Theorem 3.2 of loc.cit.; this is the version of Rubin's formula we refer to in the abstract). Rubin uses in [Rub94] the definition of [PR92] for $p$-adic height pairings. We finally note that Rubin's formula [Rub94]. Theorem 3.2, has been generalized by Howard [How04], Theorem 3.4, for abelian varieties (resp., by Nekovár in $§ 11.5 .10$ of [Nek06] for general motives) whose $L$-functions vanish to higher order. We provide an overview of Rubin's formula since it is one of the main motivations for the results of the current paper.

Suppose $E_{/ \mathbb{Q}}$ is an elliptic curve which has good, ordinary reduction at $p$. Let $\mathbb{Q}_{\infty}$ be the unique $\mathbb{Z}_{p}$-extension of $\mathbb{Q}$, and for every $n$, let $\mathbb{Q}_{n}$ be the unique sub-extension of $\mathbb{Q}$ of degree $p^{n}$. Put $\Phi_{n}=\mathbb{Q}_{n} \otimes \mathbb{Q}_{p}$ and $\Phi_{\infty}=\cup \Phi_{n}$. Let $T_{p}(E)$ denote the $p$ adic Tate module of $E$, and suppose we are given a sequence of cohomology classes $\mathbf{z}=\left\{z_{n}\right\} \in \lim H^{1}\left(\mathbb{Q}_{n}, T_{p}(E)\right)$. Using local Tate cup-product pairing, one obtains an element $\overleftarrow{f_{\mathbf{z}}} \in \operatorname{Hom}\left(E\left(\Phi_{\infty}\right), \mathbb{Z}_{p}\right)$; see equation (5) of [Rub94]. The following is Theorem 3.2 (i) of loc.cit.:

Theorem (Rubin). Let $S_{p}(E / \mathbb{Q})$ denote the p-adic Selmer group of $E_{\mathbb{Q}}$ over $\mathbb{Q}$. Then $z_{0} \in S_{p}(E / \mathbb{Q})$ if and only if $f_{\mathbf{z}}\left(E\left(\mathbb{Q}_{p}\right)\right)=0$.

When $f_{\mathbf{z}}\left(E\left(\mathbb{Q}_{p}\right)\right)=0$, Rubin constructs in $\S 3$ of [Rub94] a derivative $\operatorname{Der}_{\rho}\left(f_{\mathbf{z}}\right)$ of $f_{\mathbf{z}}$ along $\rho$, where $\rho$ is any nonzero homomorphism $\operatorname{Gal}\left(\mathbb{Q}_{\infty} / \mathbb{Q}\right) \longrightarrow \mathbb{Z}_{p}$. See also the remarks preceding Theorem 3.2 and Proposition 7.1 of [Rub94]. Rubin's formula can be stated as follows:

Theorem (Rubin). Suppose $z_{0} \in S_{p}(E / \mathbb{Q}) \subset H^{1}\left(\mathbb{Q}, T_{p}(E)\right)$. Then for every 
$x \in E(\mathbb{Q}) \otimes \mathbb{Z}_{p}$,

$$
\left\langle z_{0}, x\right\rangle_{\rho}=\operatorname{Der}_{\rho}\left(f_{\mathbf{z}}\right)(x),
$$

where $\langle,\rangle_{\rho}$ is the $p$-adic height pairing.

This formula should be compared to our formula stated in Theorem 5.1. Having spelled out the first link between our work and results mentioned above, let us describe our results in greater detail.

In [Nek06], Nekovár defines extended Selmer groups associated to (ordinary) Galois representations, which are strictly larger than the classical Selmer groups in the presence of an exceptional zero (in the sense of [Gre94]). He also defines $p$-adic height pairings on his extended Selmer groups. One natural question is what portion of the results above may be transferred to this new setting when an exceptional zero is present. We tackle this problem in the simplest and the most classical setting: Fixing a number field $K$, the Galois representation in consideration is $T=\mathcal{O}(1) \otimes \chi^{-1}$. Here, $\mathcal{O}$ is the ring of integers of a finite extension $\mathfrak{F}$ of $\mathbb{Q}_{p}$ and $\mathcal{O}(1)=\mathcal{O} \otimes_{\mathbb{Z}_{p}} \mathbb{Z}_{p}(1)$, where $\mathbb{Z}_{p}(1)=T_{p}\left(\mathbb{G}_{m}\right)$ is as usual the $p$-adic Tate module of the multiplicative group, and $\chi: \operatorname{Gal}(\bar{K} / K) \rightarrow \mathcal{O}^{\times}$is a non-trivial Dirichlet character with the property that $\chi(\wp)=1$ for a prime $\wp$ of $K$ lying above $p$. The Rubin-style formula we prove here (Corollary 5.7) is akin to Theorem 1 in [Rub94]. Before we state it, we introduce the necessary notation.

Suppose in this introduction that $K=\mathbb{Q}$ and $\chi$ is an even Dirichlet character. See $\S 6.3$ below for the case when $K$ is a general totally real number field but $\chi$ is totally odd, and $\S 6.4$ when the base field $K$ is totally imaginary. Let $L$ be the field cut by the Dirichlet character $\chi$, i.e., the fixed field of $\operatorname{ker}(\chi)$. Let $c_{1}^{\chi} \in \widetilde{H}_{f}^{1}(\mathbb{Q}, T)$ be tame cyclotomic unit inside of $L$ defined as in $\$ 6.1$ of [MR04], see also $\$ 3$ below for a recap. Here (and below) $\widetilde{H}_{f}^{1}(K, T)$ stands for the extended Selmer groups of Nekovár; for an overview (and explicit calculations specific to our case of interest, including a description of how we view the cyclotomic units as elements of the extended Selmer groups) see $\S 2.1$ and $\S 3$ below. Set $T^{*}=\operatorname{Hom}(T, \mathcal{O}(1))=\mathcal{O}(\chi)$. Let $\langle,\rangle_{\text {Nek }}$ denote Nekovár's $p$-adic height pairing, see [Nek06], $\S 11$, for a general definition, and also $\$ 2$ below for the portion of the theory that concerns us. Attached to an arbitrary element $\alpha \in \widetilde{H}_{f}^{1}\left(\mathbb{Q}, T^{*}\right)$ and the collection of cyclotomic units $\xi$ along the cyclotomic $\mathbb{Z}_{p}$-tower, we construct a ' $p$-adic $L$-function' $L_{\xi, \Phi}$ in $\$ 5$ below. The Rubin-style formula we prove reads as follows:

Theorem A (Corollary 5.7 below). $\left\langle c_{1}^{\chi}, \alpha\right\rangle_{\mathrm{Nek}}=L_{\xi, \Phi}^{\prime}(\mathbf{1})$.

Here, 1 is the trivial character and $L_{\xi, \Phi}^{\prime}$ is the derivative of $L_{\xi, \Phi}$ along the cyclotomic character, see $\$ 5$ for details. Using Coleman's map, one may choose a particular $\Phi$ and $\alpha$, and apply Theorem A above to prove: 
Theorem B (Theorem 6.7 below). $\left\langle c_{1}^{\chi}, \operatorname{cor}_{0}^{\chi}\right\rangle_{\mathrm{Nek}}=\widetilde{L}_{p}^{\prime}(1, \chi)$.

Here, $\operatorname{col}_{0}^{\chi} \in \tilde{H}_{f}^{1}\left(\mathbb{Q}, T^{*}\right)$ is the element we obtain from Coleman's homomorphism and $\widetilde{L}_{p}(s, \chi)$ is an imprimitive Kubota-Leopoldt $p$-adic $L$-function. See $\S 6.2$ for details. See also $\$ 6.4$ for the version of this result when the base field is a quadratic imaginary number field. We remark that our formula above recovers a $p$-adic variant of Kronecker's limit formula with a new perspective offered by Nekovár's theory.

In $\S 6.3$, we present similar results for totally odd characters $\chi$ (when the base field $\mathrm{K}$ is totally real). We remark for now that when $K$ is an arbitrary totally real number field and $\chi$ is totally odd, our calculations provide a new interpretation for Gross' conjecture (and for the Ferrero-Greenberg theorem when $k=\mathbb{Q}$ ). See Theorem 6.9 and Remark 6.12 below.

See also Remark 6.14 for a related observation when the Galois representation in question is the $p$-adic Tate-module of an elliptic curve $E_{/ \mathbb{Q}}$ which has splitmultiplicative reduction at $p$.

The layout of the paper is as follows: In Section 2 we give an overview of Nekovář's theory of Selmer complexes and $p$-adic height pairings. We explicitly describe these objects in $\$ 2.2$ in the cases of interest. In sections 3-5 we restrict our attention to the case $K=\mathbb{Q}$ and $\chi$ even, and to the case when the base field $K$ is totally imaginary. In Section 3, we define three types of cyclotomic $(p-)$ units which our calculations rely on. In Section 4 , we calculate the $p$-adic height pairing on these different types of cyclotomic "units", and use our computations in Section 5 to prove a Rubin-style formula. In $\S 6$, we use this formula to compute the leading coefficients of certain $p$-adic $L$-functions in terms of Nekovář's heights.

We remark that the results of this paper are not covered by Nekováŕs's [Nek06] general treatment (e.g., by his variant of Rubin's formula in $\$ 11.3 .15$ and $\S 11.5 .10$; nor by his calculations in $\$ 11.4 .8$ ). In particular, Remark 11.4.10 in [Nek06] is erroneous. It would be of interest to extend the formalism developed in [Nek06], $\S 11.4$, to cover our setting.

A line of apology: We gave a very detailed and long outline of prior results of 'Gross-Zagier type', although the conclusions of the current paper only concern a very particular (and simple) Galois representation. This is mainly because of the author's desire to translate/transform the results in other settings into the context of [Nek06].

Acknowledgements. The author wishes to thank Ralph Greenberg and Tadashi Ochiai for helpful discussions; Karl Rubin for helpful correspondence and David Burns for an informative conversation on the results of this paper. He also is grateful to Masato Kurihara for explaining the author a related result he proved in a different setting. Special thanks are due to Jan Nekovár for his encouragement and for many 
enlightening discussions. The author started his work on this project while he was supported by a William Hodge Postdoctoral Fellowship at IHÉS and the final form of this paper was written up during his stay at Max Planck Institut für Mathematik. The author thanks both these institutes for their hospitality. The author also thanks the anonymous referee for pointing out several inaccuracies in an earlier version. This research was partially supported by the grant EU-FP7 IRG-230668 and TÜBITTAK.

1.1. Notation and hypotheses. Fix once and for all a rational prime $p>2$. For a number field $K$, write $G_{K}$ for the absolute Galois group $\operatorname{Gal}(\bar{K} / K)$. Let $\mathcal{O}$ be the ring of integers of a finite extension $\mathfrak{F}$ of $\mathbb{Q}_{p}$, and let $\chi$ denote a non-trivial Dirichlet character

$$
\chi: G_{K} \longrightarrow \mathcal{O}^{\times},
$$

which has prime-to- $p$ order and which satisfies $\chi(\wp)=1$ for a prime $\wp \subset K$ lying above $p$. In this paper, we will only ${ }^{1}$ deal with the case $K=\mathbb{Q}$ or $K=k$, where $k$ is a quadratic imaginary number field such that the prime $p$ splits in $k / \mathbb{Q}$.

Define $T=\mathcal{O}(1) \otimes \chi^{-1}$ and $T^{*}=\mathcal{O}(\chi)$, rank one $\mathcal{O}$-modules with a $G_{K}$-action. Here $\mathcal{O}(1)$ is the Tate twist.

Let $L$ will be the fixed field of $\operatorname{ker}(\chi)$ and let $\Delta=\operatorname{Gal}(L / K)$. Our assumption that $\chi(\wp)=1$ is equivalent to saying that $\wp$ splits completely in $L / K$. Let $S_{\wp}=\{v \mid \wp\}$ denote the collection of places of $L$ above $\wp$ (the letter " $v$ " is reserved to stand for these places of $L$ ), and let $L_{v}$ denote the completion of $L$ at $v$. Although $L_{v}=K_{\wp}$ for each $v$, we will distinguish the completions of $L$ at different places (as different embeddings $\left.L \hookrightarrow \overline{\mathbb{Q}}_{p}\right)$ and set $G_{v}=\operatorname{Gal}\left(\overline{\mathbb{Q}}_{p} / L_{v}\right)$ for a fixed algebraic closure $\overline{\mathbb{Q}}_{p}$ of $\mathbb{Q}_{p}$.

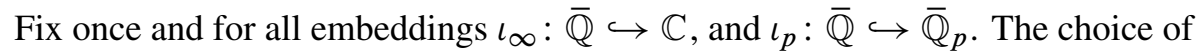
$\iota_{p}$ fixes a prime $v_{0} \in S_{\wp}$.

Let $\mathbb{Q} \infty / \mathbb{Q}$ denote the cyclotomic $\mathbb{Z}_{p}$-extension of $\mathbb{Q}$ and let $\Gamma=\operatorname{Gal}\left(\mathbb{Q}_{\infty} / \mathbb{Q}\right)$. We write $\rho_{\text {cyc }}$ for the cyclotomic character $\rho_{\text {cyc }}: \Gamma \stackrel{\sim}{\rightarrow} 1+p \mathbb{Z}_{p}$. Let $\mathbb{Q}_{n}$ denote the unique sub-extension of $\mathbb{Q}_{\infty} / \mathbb{Q}$ of degree $p^{n}$ over $\mathbb{Q}$, i.e., the fixed field of $\Gamma^{p^{n}}$. Let $\Phi_{n}$ be the completion of $\mathbb{Q}_{n}$ at the unique prime of $\mathbb{Q}_{n}$ above $p$, and set $\Phi_{\infty}=\cup \Phi_{n}$, the cyclotomic $\mathbb{Z}_{p}$-extension of $\mathbb{Q}_{p}$. By slight abuse of notation $\operatorname{Gal}\left(\Phi_{\infty} / \mathbb{Q}_{p}\right)$ will be denoted by $\Gamma$ as well. We fix a topological generator $\gamma$ of $\Gamma$. We also set $\Lambda=\mathcal{O}[[\Gamma]$ as the cyclotomic Iwasawa algebra.

When the base field $K$ is the quadratic imaginary number field $k$ which satisfies the assumption that $p$ splits in $k / \mathbb{Q}$, we write $p=\wp \wp^{*}$ with $\wp \neq \wp^{*}$. Also in this case, we assume that $p$ does not divide the class number $h_{k}$ of $k$. For an $\mathcal{O}_{k}$-ideal $\mathfrak{I}$, let $k(\mathfrak{I})$ be the ray class field of conductor $\mathfrak{I}$. For each $n \geq 0$ we write

$$
\operatorname{Gal}\left(k\left(\wp^{n+1}\right) / k\right)=\operatorname{Gal}\left(k\left(\wp^{n+1}\right) / k(\wp)\right) \times H,
$$

\footnotetext{
${ }^{1}$ Except in Remark 6.12, where we say how the arguments of $\$ 6.3$ apply for a general totally real number field.
} 
where $H$ is isomorphic to $\operatorname{Gal}(k(\wp) / k)$ by restriction. We set

$$
k_{n}=k\left(\wp^{n+1}\right)^{H}, \quad k_{\infty}=\bigcup_{n \geq 0} k_{n} .
$$

Then $k_{\infty} / k$ is a $\mathbb{Z}_{p}$-extension and we write $\Gamma:=\operatorname{Gal}\left(k_{\infty} / k\right)$ also when there is no danger of confusion. The extension $k_{\infty} / k$ is the unique $\mathbb{Z}_{p}$-extension which is unramified outside $\wp$. The prime $\wp$ is totally ramified in $k_{\infty} / k$. Let $f_{L} \subset \mathcal{O}_{k}$ denote the conductor of $L$ (which is prime to $\wp$ by our assumptions on $\chi$ ) and let $f$ be a multiple of $\mathfrak{f}_{L}$ which is prime to $\wp$ and which also satisfies the condition that the map $\mathcal{O}_{k}^{\times} \rightarrow\left(\mathcal{O}_{k} / \mathfrak{f}\right)^{\times}$is injective. Attached to a Grössencharacter $\varphi$ of $k$ of infinity type $(1,0)$ and of conductor $\mathfrak{f}$, there is an elliptic curve $E$ defined over $F=k(\mathfrak{f})$ with the properties that

- $E$ has complex multiplication by $\mathcal{O}_{k}$

- $F\left(E_{\text {tor }}\right)$ is an abelian extension of $k$,

where we write $F\left(E_{\text {tor }}\right)$ for the extension of $K$ which is generated by the coordinates of the torsion-submodule $E_{\text {tor }} \subset E(\bar{k})$. For such $E$, we have $F\left(E\left[\wp^{n+1}\right]\right)=$ $k\left(\mathfrak{F} \wp^{n+1}\right)$ for all $n \geq 0$, and using this fact one obtains a canonical identification $\operatorname{Gal}\left(F\left(E\left[\wp^{\infty}\right]\right) / F(E[\wp])\right) \stackrel{\sim}{\rightarrow} \Gamma$ and the following isomorphisms:

(i) $\rho_{E}: \operatorname{Gal}\left(F\left(E\left[\wp^{\infty}\right]\right) / F\right) \stackrel{\sim}{\longrightarrow} \operatorname{Aut}\left(E\left[\wp^{\infty}\right]\right)=\mathcal{O}_{k_{\wp}}^{\times} \stackrel{\sim}{\longrightarrow} \mathbb{Z}_{p}^{\times}$,

(ii) $\rho_{\Gamma}:=\left.\rho_{E}\right|_{\Gamma}: \Gamma \stackrel{\sim}{\longrightarrow} 1+p \mathbb{Z}_{p}$.

The character $\rho_{\Gamma}$ will play the role of cyclotomic character when our base field $K$ is the quadratic imaginary number field $k$.

For any finitely generated abelian group $M$ endowed with a $G_{K}$ action, $\widehat{M}$ will denote its $p$-adic completion $\operatorname{Hom}\left(\operatorname{Hom}\left(M, \mathbb{Q}_{p} / \mathbb{Z}_{p}\right), \mathbb{Q}_{p} / \mathbb{Z}_{p}\right)$, and $M^{\chi}$ will denote the $\chi$-isotypic part of $\hat{M} \otimes_{\mathbb{Z}_{p}} \mathcal{O}$. Also, let $\log _{p}: 1+p \mathbb{Z}_{p} \rightarrow \mathbb{Z}_{p}$ denote the $p$-adic logarithm.

For a field $K$ (with fixed separable closure $\bar{K} / K$ ) and a $\mathcal{O}[[\mathrm{Gal}(\bar{K} / K)]$-module $X$ which is finitely generated over $\mathcal{O}$, we will denote the $i$-th cohomology (with continuous cochains) of the group $\operatorname{Gal}(\bar{K} / K)$ with coefficients in $X$ by $H^{i}(K, X)$.

For every positive integer $n$, we define $\mu_{n} \subset \overline{\mathbb{Q}}$ to be the set of $n$th roots of unity.

\section{Height pairings on extended Selmer groups}

2.1. Generalities. In this section we very briefly review Nekovár's theory of Selmer complexes and his definition of extended Selmer groups. The treatment in this section is far more general than what is needed for the purposes of this paper, and it is much less general than what is covered in [Nek06]. For example, we focus on coefficient 
rings such as the ring of integers $\mathcal{O}$ of a finite extension of $\mathbb{Q}_{p}$, or the one variable Iwasawa algebra $\mathcal{O}[[\Gamma]$; and we restrict our attention to a complex of $\mathcal{O}$-modules $M$ of finite type, endowed with a continuous action of the absolute Galois group $G_{K}$ of a fixed base field $K$, concentrated in degree zero. From $\$ 2.2$ on, $K$ will be $\mathbb{Q}$ (except in $\$ 6.4$ where $K=k$, a quadratic imaginary number field and Remark 6.12 where $K$ is an arbitrary totally real field), and $M$ will be one of $\mathcal{O}(1) \otimes \chi^{-1}, \mathcal{O}(\chi), \mathcal{O}(1)$ or $\mathcal{O}$ (in degree zero) .

Let $G$ be a profinite group (given the profinite topology) and let $\mathcal{O}$ be as above. Let $M$ be a free $\mathcal{O}$-module of finite type on which $G$ acts continuously. Then $M$ is admissible in the sense of [Nek06] (see \$3.2), and we can talk about the complex of continuous cochains $C^{\bullet}(G, M)$ as in $\$ 3.4$ of loc.cit. Let $K$ be a number field with a fixed algebraic closure $\bar{K}$ and let $S$ denote a finite set of primes of $K$ which contains all primes above $p$, all primes at which the representation $M$ is ramified and all infinite places of $K$, let $S_{f}$ denote the subset of finite places of $S$. Let $K_{S}$ the maximal sub-extension of $\bar{K} / K$ which is unramified outside $S$, and let $G_{K, S}$ denote the Galois group $\operatorname{Gal}\left(K_{S} / K\right)$. For all $w \in S_{f}$, we write $K_{w}$ for the completion of $K$ at $w$, and $G_{w}$ for its absolute Galois group. Whenever it is convenient, we will identify $G_{w}$ with a decomposition subgroup inside $G_{K}:=\operatorname{Gal}(\bar{K} / K)$. We will be interested in the cases $G=G_{K, S}$ or $G=G_{w}$.

2.1.1. Selmer complexes. Classical Selmer groups are defined as elements of the global cohomology group $H^{1}\left(G_{K, S}, M\right)$ satisfying certain local conditions; see $\S 2.1$ of [MR04] for the most general definition. The main idea of [Nek06] is to impose local conditions on the level of complexes. We go over basics of Nekovár's theory, for details see [Nek06].

Definition 2.1. Local conditions for $M$ are given by a collection

$$
\Delta(M)=\left\{\Delta_{w}(M)\right\}_{w \in S_{f}}
$$

where $\Delta_{w}(M)$ stands for a morphism of complexes of $\mathcal{O}$-modules

$$
i_{w}^{+}(M): U_{w}^{+} \longrightarrow C^{\bullet}\left(G_{w}, M\right)
$$

for each $w \in S_{f}$.

Also set

$$
U_{v}^{-}(M)=\operatorname{Cone}\left(U_{v}^{+}(M) \stackrel{-i_{v}^{+}}{\longrightarrow} C^{\bullet}\left(G_{v}, M\right)\right)
$$

and

$$
U_{S}^{ \pm}(M)=\bigoplus_{w \in S_{f}} U_{w}^{ \pm}(M) ; \quad i_{S}^{+}(M)=\left(i_{w}^{+}(M)\right)_{w \in S_{f}}
$$


We also define

$$
\operatorname{res}_{S_{f}}: C^{\bullet}\left(G_{K, S}, M\right) \longrightarrow \bigoplus_{w \in S_{f}} C^{\bullet}\left(G_{w}, M\right)
$$

as the canonical restriction morphism.

Definition 2.2. The Selmer complex associated with the choice of local conditions $\Delta(M)$ on $M$ is given by the complex

$$
\begin{aligned}
& \widetilde{C}_{f}^{\bullet}\left(G_{K, S}, M, \Delta(M)\right) \\
& \quad:=\operatorname{Cone}\left(C^{\bullet}\left(G_{K, S}, M\right) \bigoplus U_{S}^{+}(M) \stackrel{\mathrm{res}_{S_{f}}{ }^{-i_{S}^{+}}(M)}{\longrightarrow} \bigoplus_{w \in S_{f}} C^{\bullet}\left(G_{w}, M\right)\right)[-1]
\end{aligned}
$$

where $[n]$ denotes a shift by $n$. The corresponding object in the derived category will be denoted by $\tilde{\mathbf{R}} \Gamma_{f}\left(G_{K, S}, M, \Delta(M)\right)$ and its cohomology by $\tilde{H}_{f}^{i}\left(G_{K, S}, M, \Delta(M)\right)$ (or simply by $\tilde{H}_{f}^{i}(K, M)$ or by $\tilde{H}_{f}^{i}(M)$ when there is no danger of confusion). The $\mathcal{O}$-module $\widetilde{H}_{f}^{1}(M)$ will be called the extended Selmer group.

The object in the derived category corresponding to the complex $C^{\bullet}\left(G_{K, S}, M\right)$ will be denoted by $\mathbf{R} \Gamma\left(G_{K, S}, M\right)$.

2.1.2. Comparison with classical Selmer groups. For each $w \in S_{f}$, suppose that we are given a submodule

$$
H_{\mathscr{F}}^{1}\left(K_{w}, M\right) \subset H^{1}\left(K_{w}, M\right) .
$$

This data which $\mathcal{F}$ encodes is called a Selmer structure on $M$. Starting with $\mathcal{F}$, one defines the Selmer group as

$$
H_{\mathscr{F}}^{1}(K, M):=\operatorname{ker}\left\{H^{1}\left(G_{K, S}, M\right) \longrightarrow \bigoplus_{w \in S_{f}} \frac{H^{1}\left(K_{w}, M\right)}{H_{\mathscr{F}}^{1}\left(K_{w}, M\right)}\right\} .
$$

On the other hand, as explained in $§ 6.1 .3 .1-\S 6.1 .3 .2$ of [Nek06], there is an exact triangle

$$
U_{S}^{-}(M)[-1] \longrightarrow \widetilde{\mathbf{R} \Gamma}{ }_{f}\left(G_{K, S}, M, \Delta(M)\right) \longrightarrow \mathbf{R} \Gamma\left(G_{K, S}, M\right) \longrightarrow U_{S}^{-}(M)
$$

This gives rise to an exact sequence in the level of cohomology:

Proposition 2.3 ([Nek06], §0.8.0 and §9.6). For each $i$, the following sequence is exact:

$\cdots \longrightarrow H^{i-1}\left(U_{S}^{-}(M)\right) \longrightarrow \tilde{H}_{f}^{i}(M) \longrightarrow H^{i}\left(G_{K, S}, M\right) \longrightarrow H^{i}\left(U_{S}^{-}(M)\right) \longrightarrow \cdots$ 
This proposition is used to compare Nekováŕ's extended Selmer groups to classical Selmer groups. Although this may be achieved in greater generality, we will only state the relevant comparison theorem for Greenberg's local conditions (and Greenberg's Selmer groups) whose definitions we now recall. For further details, see [Gre89], [Gre94], [Nek06].

Let $I_{w}$ denote the inertia subgroup of $G_{w}$. Suppose we are given an $\mathcal{O} \llbracket\left[G_{w} \rrbracket\right]-$ submodule $M_{w}^{+}$of $M$ for each place $w \mid p$ of $K$, set $M_{w}^{-}=M / M_{w}^{+}$. Then Greenberg's local conditions (on the complex level, i.e., in the sense of [Nek06], §6) are given by

$$
U_{w}^{+}= \begin{cases}C^{\bullet}\left(G_{w}, M_{w}^{+}\right) & \text {if } w \mid p, \\ C^{\bullet}\left(G_{w} / I_{w}, M^{I_{w}}\right) & \text { if } w \nmid p\end{cases}
$$

with the obvious choice of morphisms

$$
i_{w}^{+}(M): U_{w}^{+}(M) \longrightarrow C^{\bullet}\left(G_{w}, M\right) .
$$

As in Definition 2.2, we then obtain a Selmer complex and an extended Selmer group, which we denote by $\widetilde{H}_{f}^{1}(M)$. Greenberg's local conditions are the only type of local conditions we will deal with from now on.

We now define the relevant Selmer structure ${ }^{2} \mathcal{F}_{\text {can }}$ on $M$.

Definition 2.4. The canonical Selmer structure $\mathcal{F}_{\text {can }}$ is given by

$$
H_{\mathcal{F}_{\text {can }}}^{1}\left(K_{w}, M\right)= \begin{cases}\operatorname{im}\left(H^{1}\left(G_{w}, M_{w}^{+}\right) \rightarrow H^{1}\left(K_{w}, M\right)\right) & \text { if } w \mid p, \\ =\operatorname{ker}\left(H^{1}\left(G_{w}, M_{w}\right) \rightarrow H^{1}\left(G_{w}, M_{w}^{-}\right)\right) & \\ \operatorname{ker}\left(H^{1}\left(G_{w}, M\right) \rightarrow H^{1}\left(I_{w}, M\right)\right) & \text { if } w \nmid p . \\ =\operatorname{im}\left(H^{1}\left(G_{w} / I_{w}, M^{I_{w}}\right) \rightarrow H^{1}\left(G_{w}, M\right)\right) & \end{cases}
$$

Hence, we obtain the following Selmer group (which is called the strict Selmer group in [Nek06], §9.6.1, and denoted by $\left.S_{M}^{\mathrm{str}}(K)\right)$ :

$$
H_{\mathcal{F}_{\text {can }}}^{1}(K, M)=\operatorname{ker}\left(H^{1}\left(G_{K, S}, M\right) \longrightarrow \bigoplus_{w \mid p} H^{1}\left(G_{w}, M_{w}^{-}\right) \oplus \bigoplus_{w \nmid p} H^{1}\left(I_{w}, M\right)\right) .
$$

Proposition 2.3 now shows that:

Proposition 2.5. The following sequence is exact:

$$
M^{G_{K}} \longrightarrow \bigoplus_{w \mid p}\left(M_{w}^{-}\right)^{G_{w}} \longrightarrow \tilde{H}_{f}^{1}(M) \longrightarrow H_{\mathcal{F}_{\text {can }}}^{1}(K, M) \longrightarrow 0 .
$$

\footnotetext{
${ }^{2}$ For a general $\boldsymbol{M}$, our definition of $\mathcal{F}_{\text {can }}$ (the canonical Selmer structure) slightly differs from its original definition in [MR04]. However, for the specific Galois representation we use starting from $\$ 2.2$ on, they do coincide.
} 
See [Nek06], Lemma 9.6.3, for a proof.

Remark 2.6. Note that if $\left(M_{w}^{-}\right)^{G_{w}}=0$ for all $w \mid p$, then the extended Selmer group $\widetilde{H}_{f}^{1}(M)$ coincides with the canonical Selmer group $H_{\widetilde{F}_{\text {can }}}^{1}(K, M)$. However, if some $\left(M_{w}^{-}\right)^{G_{w}} \neq 0$ then $\tilde{H}_{f}^{1}(M)$ is strictly larger than $H_{\mathcal{F}_{\text {can }}}^{1}(K, M)$ (under the assumption that $M^{G_{K}}=0$, say). This is the main feature of Nekovářr's Selmer complexes: They reflect the existence of exceptional zeros, unlike classical Selmer groups.

2.1.3. Height pairings. We now recall Nekováŕ's definition of height pairings on his extended Selmer groups. All the references in this section are to $\$ 11$ of [Nek06] unless otherwise stated.

Let $M^{*}=\operatorname{Hom}(M, \mathcal{O})(1)$ (in Nekováŕ's language this is $\mathcal{D}(M)(1)$, the Grothendieck dual of $M$ ). Let $\Gamma$ be the Galois group $\operatorname{Gal}\left(\mathbb{Q}_{\infty} / \mathbb{Q}\right.$ ) (resp., the Galois group $\left.\operatorname{Gal}\left(k_{\infty} / k\right)\right)$ and $\rho$ be the cyclotomic character $\rho_{\text {cyc }}$ (resp., the character $\rho_{\Gamma}$ ) when the base field $K$ is $\mathbb{Q}$ (also more generally, when $K$ is a totally real number field) (resp., when $K$ is the quadratic imaginary number field $k$ ). The height pairing

$$
\langle,\rangle_{\mathrm{Nek}}: \tilde{H}_{f}^{1}(M) \otimes_{\mathcal{O}} \tilde{H}_{f}^{1}\left(M^{*}\right) \longrightarrow \mathcal{O} \otimes_{\mathbb{Z}_{p}} \Gamma \stackrel{\mathrm{id} \otimes \log _{p} \rho}{\longrightarrow} \mathcal{O}
$$

is defined in two steps:

(i) Apply the Bockstein morphism

$$
\beta: \widetilde{\mathbf{R}} \Gamma_{f}(M) \longrightarrow \widetilde{\mathbf{R}}_{f}(M)[1] \otimes_{\mathbb{Z}_{p}} \Gamma \stackrel{\mathrm{id} \otimes \log _{p} \rho}{\longrightarrow} \widetilde{\mathbf{R}}_{f}(M)[1]
$$

See $\$ 11.1 .3$ in [Nek06] for the original definition of $\beta$. Let $\beta^{1}$ denote the map induced on the level of cohomology:

$$
\beta^{1}: \tilde{H}_{f}^{1}(M) \longrightarrow \tilde{H}_{f}^{2}(M) .
$$

(ii) Use the global duality pairing

$$
\langle,\rangle_{\mathrm{PT}}: \tilde{H}_{f}^{2}(M) \otimes_{\mathcal{O}} \tilde{H}_{f}^{1}\left(M^{*}\right) \longrightarrow \mathcal{O}
$$

on the image of $\beta^{1}$ inside of $\tilde{H}_{f}^{2}(M)$. Here the subscript PT stands for PoitouTate, and the global pairing comes from summing up the invariants of the local cup product pairing, see $\S 6.3$ in [Nek06] for more details.

Just as for other height pairings, universal norms are in the kernel of Nekovář's height pairing: 
Proposition 2.7 ([Nek06], Proposition 11.5.7 and §11.5.8). For $X=M, M^{*}$, the universal norms

$$
\operatorname{im}\left(\tilde{H}_{f}^{1}\left(G_{K, S}, X \otimes_{\mathcal{O}} \mathcal{O}[[\Gamma]], \Delta(M) \otimes \mathcal{O}[[\Gamma]]\right) \longrightarrow \tilde{H}_{f}^{1}(X)\right)
$$

are in the kernel of the height pairing $\langle,\rangle_{\mathrm{Nek}}$.

Here $\Delta(M) \otimes \mathcal{O} \llbracket \Gamma \rrbracket$ stands for an appropriate propagation of the local conditions $\Delta(M)$ on $M$ to $M \otimes_{\mathcal{O}} \mathcal{O}[[\Gamma]]$, see $\S 8$ of [Nek06] (particularly $\S 8.6$ ) for details.

2.2. The classical case: $\boldsymbol{T}=\boldsymbol{\mathcal { O }}(\mathbf{1}) \otimes \chi^{\mathbf{- 1}}$. In this section we explicitly calculate both the classical Selmer groups and the extended Selmer groups associated with the representations $T=\mathcal{O}(1) \otimes \chi^{-1}$ and $T^{*}=\mathcal{O}(\chi)$, viewed as a representation of $G_{K}$. We keep the notation of $\S 2.1$. Let $S=\left\{\mathfrak{q}: \mathfrak{q} \mid p \mathfrak{f}_{\chi} \infty\right\}$ be a set of places of $K$. We set $T^{+}=T,\left(T^{*}\right)^{+}=0$ (hence $\left.T^{-}=0,\left(T^{*}\right)^{-}=T^{*}\right)$.

Lemma 2.8. (i) $\widetilde{H}_{f}^{1}(K, T) \stackrel{\sim}{\longrightarrow} H_{\mathscr{F}_{\text {can }}}^{1}(K, T)$.

(ii) The sequence

$$
0 \longrightarrow \bigoplus_{\wp \mid p} H^{0}\left(K_{\wp}, \mathcal{O}(\chi)\right) \longrightarrow \tilde{H}_{f}^{1}\left(K, T^{*}\right) \longrightarrow H_{\mathcal{F}_{\text {can }}}^{1}\left(K, T^{*}\right) \longrightarrow 0
$$

is exact.

Proof. Immediate from Proposition 2.5.

Remark 2.9. For our particular Galois representation $T$, the Selmer group $H_{\mathscr{F}_{\text {can }}}^{1}(K, T)$ as defined above agrees with what [MR04] calls $H_{\mathscr{F}_{\text {can }}}^{1}(K, T)$. Indeed, in the language of [MR04], $H_{\mathcal{F}_{\text {can }}}^{1}(\mathbb{Q}, T)$ is defined as

$$
H_{\mathcal{F}_{\text {can }}}^{1}(K, T)=\operatorname{ker}\left(H^{1}\left(G_{K, S}, T\right) \longrightarrow \bigoplus_{\mathfrak{q} \in S, \mathfrak{q} \nmid p} \frac{H^{1}\left(K_{\mathfrak{q}}, T\right)}{H_{f}^{1}\left(K_{\mathfrak{q}}, T\right)}\right)
$$

where $\mathfrak{f}=\mathfrak{f}_{\chi}$ denotes the conductor of $\chi$, and $H_{f}^{1}\left(K_{\mathfrak{q}}, T\right) \subset H^{1}\left(K_{\mathfrak{q}}, T\right)$ is as in [Rub00], Definition I.3.4. Let

$$
H_{\mathrm{ur}}^{1}\left(K_{\mathfrak{q}}, T\right)=\operatorname{ker}\left(H^{1}\left(K_{\mathfrak{q}}, T\right) \longrightarrow H^{1}\left(I_{\mathfrak{q}}, T\right)\right) .
$$

It follows from Lemma I.3.5 (iii) in [Rub00] that

$$
H_{f}^{1}\left(K_{\mathfrak{q}}, T\right)=H_{\mathrm{ur}}^{1}\left(K_{\mathfrak{q}}, T\right)
$$


for every $\mathfrak{q} \nmid p$ (including primes $\mathfrak{q} \mid \mathfrak{f}_{\chi}$ ), hence it follows that the canonical Selmer group of [MR04] is given by

$$
H_{\mathscr{F}_{\text {can }}}^{1}(K, T)=\operatorname{ker}\left(H^{1}\left(G_{K, S}, T\right) \longrightarrow \bigoplus_{\mathfrak{q} \in S, \mathfrak{q} \nmid p} H^{1}\left(I_{\mathfrak{q}}, T\right)\right) .
$$

This shows that our definition of the canonical Selmer group given by (2.1) agrees with the definition of [MR04].

Proposition 2.10. Let $\mathcal{O}_{L}$ denote the ring of integers of $L, \mathcal{O}_{L}[1 / p]$ its $p$-integers, $\mathcal{O}_{L}^{\times}$its unit group and $\mathcal{O}_{L}[1 / p]^{\times}$its p-units.

(i) $H_{\mathscr{F}_{\text {can }}}^{1}(K, T)=\left(\mathcal{O}_{L}[1 / p]^{\times}\right)^{\chi}$,

(ii) $H_{\mathscr{F}_{\text {can }}}^{1}\left(K, T^{*}\right)=0$.

Proof. The first part follows from Remark 2.9 and [MR04], Equation (25). For the second part, observe that $H_{\mathscr{F}_{\text {can }}}^{1}\left(\mathbb{Q}, T^{*}\right)$ is contained in the submodule of unramified homomorphisms inside

$$
H^{1}\left(K, T^{*}\right)=\operatorname{Hom}\left(G_{L}, \mathcal{O}\right)^{\chi^{-1}}
$$

where the equality is obtained from the inflation-restriction sequence. In other words,

$$
H_{\mathscr{F}_{\text {can }}}^{1}\left(K, T^{*}\right) \subset \operatorname{Hom}\left(\operatorname{Gal}\left(H_{L} / L\right), \mathcal{O}\right)^{\chi^{-1}}
$$

where $H_{L}$ denotes the Hilbert class field of $L$. But since $\operatorname{Gal}\left(H_{L} / L\right)$ is finite, we have $\operatorname{Hom}\left(\operatorname{Gal}\left(H_{L} / L\right), \mathcal{O}\right)=0$, so $H_{\mathscr{F}_{\text {can }}}^{1}\left(K, T^{*}\right)=0$ as well.

Corollary 2.11. Keep the notation above.

(i) $\tilde{H}_{f}^{1}(K, T)=\left(\mathcal{O}_{L}[1 / p]^{\times}\right)^{\chi}$,

(ii) $\bigoplus_{\wp \mid p} H^{0}\left(K_{\wp}, \mathcal{O}(\chi)\right) \stackrel{\sim}{\longrightarrow} \tilde{H}_{f}^{1}\left(K, T^{*}\right)$.

We suppose until the end of this paper that

(H) $\chi(\wp)=1$ for a prime $\wp \subset K$ lying above $p$, and that $\chi\left(\wp^{\prime}\right) \neq 1$ for any other $\wp^{\prime} \subset K$ above $p$.

It follows from Corollary 2.11 that $\tilde{H}_{f}^{1}\left(\mathbb{Q}, T^{*}\right)$ is a free $\mathcal{O}$-module of rank one. Furthermore, it follows from the proof of Proposition III.2.6 (ii) in [Rub00] that we have

$$
\left(\mathcal{O}_{L}[1 / p]^{\times}\right)^{\chi}=\left(\mathcal{O}_{L}[1 / \wp]^{\times}\right)^{\chi}
$$

since we assume $(\mathbf{H})$. 
When $K=\mathbb{Q}$ and $\chi$ is an even character, it follows from Theorem 5.2.15 in [MR04] that the core Selmer rank of the canonical Selmer structure (in the sense of Definition 4.1.11 of loc.cit., see also Corollary 5.2.6 of loc.cit.) is 2 (since we assumed $\chi$ is even and $\chi(p)=1)$; hence $H_{\widetilde{F}_{\text {can }}}^{1}(\mathbb{Q}, T)=\widetilde{H}_{f}^{1}(\mathbb{Q}, T)$ is a free $\mathcal{O}$-module of rank 2. We will later describe an explicit $\mathfrak{F}$-basis for $\widetilde{H}_{f}^{1}(\mathbb{Q}, T) \otimes \mathfrak{F}$.

When $K$ is totally real and $\chi$ is totally odd, then $\left(\mathcal{O}_{L}[1 / p]^{\times}\right)^{\chi}=\left(\mathcal{O}_{L}[1 / \wp]^{\times}\right)^{\chi}$ (resp., $\mathcal{O}_{L}^{\times, \chi}$ ) is a free $\mathcal{O}$-module of rank one (resp., of rank zero) and hence $\widetilde{H}_{f}^{1}(K, T)$ is also free of rank one.

Let $\beta_{\chi}^{1}: \widetilde{H}_{f}^{1}(\mathbb{Q}, T) \rightarrow \widetilde{H}_{f}^{2}(\mathbb{Q}, T)$ denote the Bockstein morphism, as in $\S 2.1 .3$ above.

Proposition 2.12. For any $x \in \widetilde{H}_{f}^{1}(K, T)$ and $y \in \widetilde{H}_{f}^{1}\left(K, T^{*}\right)$,

$$
\langle x, y\rangle_{\mathrm{Nek}}=\left\langle\beta_{\chi}^{1}(x), y\right\rangle_{\mathrm{PT}} .
$$

Proof. This is just a restatement of the definition of Nekovár's height pairing we gave in $\S 2.1 .3$.

\section{Cyclotomic units}

Throughout $\S 3$, our base field $K$ is $\mathbb{Q}$ and $\chi$ is an even, non-trivial Dirichlet character whose order is prime to $p$ and which has the property that $\chi(p)=1$. Let $L$ be the field cut by $\chi$ and write $\Delta:=\operatorname{Gal}(L / \mathbb{Q})$. We set $e_{\chi}:=\sum_{\delta \in \Delta} \chi^{-1}(\delta) \delta \in \mathcal{O}[\Delta]$. In this section, we define three different types of special elements which will be crucial in what follows: Tame cyclotomic units, wild cyclotomic units and Solomon's wild cyclotomic p-units defined as in [Sol92].

Fix a collection $\left\{\zeta_{m}: m \geq 1\right\}$ such that $\zeta_{m}$ is a primitive $m$-th root of unity and $\zeta_{m n}^{n}=\zeta_{m}$ for every $m$ and $n$. Let $f=f_{\chi}$ denote the conductor of $\chi$, and recall the Kummer map which induces a canonical map

$$
F^{\times} \longrightarrow H^{1}\left(F, \mathbb{Z}_{p}(1)\right)
$$

for every finite abelian extension $F$ of $\mathbb{Q}$.

Definition 3.1. For every positive integer $n$ prime to $p$, define

$$
c_{n}=\mathrm{N}_{\mathbb{Q}\left(\mu_{n f}\right) / L\left(\mu_{n}\right)}\left(\zeta_{n f}-1\right) \in L\left(\mu_{n}\right)^{\times}
$$

and

$$
c_{n}^{\chi}=e_{\chi} \mathrm{N}_{\mathbb{Q}\left(\mu_{n f}\right) / L\left(\mu_{n}\right)}\left(\zeta_{n f}-1\right) \in L\left(\mu_{n}\right)^{\times, \chi}=H^{1}\left(\mathbb{Q}\left(\mu_{n}\right), T\right) .
$$


The collection $\mathbf{c}=\left\{c_{n}^{\chi}:(n, p)=1\right\}$ is called the collection of tame $\chi$-cyclotomic units. The element $c_{1}^{\chi}$ is called the tame $\chi$-cyclotomic unit of $L$, or simply the tame cyclotomic unit once $\chi$ (thus also $L$ ) is fixed.

For every finite abelian extension $F$ of $\mathbb{Q}$ of conductor $m$, define

$$
\xi_{F}=\mathbf{N}_{\mathbb{Q}\left(\mu_{m p}\right) / F}\left(\zeta_{m p}-1\right) .
$$

Here and elsewhere in this paper, the symbol $\mathbf{N}$ stands for the norm map.

Let $\mathbb{Q}_{\infty}$ be the cyclotomic $\mathbb{Z}_{p}$-extension of $\mathbb{Q}$, and $\mathbb{Q}_{n}$ be its unique sub-extension of degree $p^{n}$ over $\mathbb{Q}$. We set $L_{n}:=L \mathbb{Q}_{n}$. Note that the collection $\left\{\xi_{F}\right\}$ satisfies the Euler system distribution relation, in particular the collection $\left\{\xi_{L_{n}}: n \geq 1\right\}$ is norm-coherent.

Definition 3.2. The collection

$$
\xi=\xi_{\infty}^{\chi}:=\left\{e_{\chi} \xi_{L_{n}}: n \geq 1\right\} \in{\underset{\leftarrow}{\lim }}_{H^{1}}\left(\mathbb{Q}_{n}, T\right)
$$

is called the wild $\chi$-cyclotomic units. When $\chi$ is understood, this collection will be called the collection of wild cyclotomic units.

3.1. Cyclotomic units and 'exceptional zeros'. From our assumption that $\chi(p)=$ 1 , it follows that $p$ splits completely in $L$.

Lemma 3.3. Under the running assumptions $\xi_{L}=1$.

Proof. This is [Sol92], Lemma 2.2; see also Remark 6.1.10 in [MR04].

Let $\Gamma=\operatorname{Gal}\left(\mathbb{Q}_{\infty} / \mathbb{Q}\right)$ and $\left.\Lambda=\mathcal{O}[\Gamma]\right]$. Let $\log _{p}: \mathbb{Z}_{p}^{\times} \rightarrow \mathbb{Z}_{p}$ be the $p$-adic logarithm, and let $\rho_{\text {cyc }}: \Gamma \rightarrow 1+p \mathbb{Z}_{p}$ be the cyclotomic character. Fix a topological generator $\gamma$ of $\Gamma$. The short exact sequence

$$
0 \longrightarrow T \otimes \Lambda \stackrel{\gamma-1}{\longrightarrow} T \otimes \Lambda \longrightarrow T \longrightarrow 0
$$

induces a long exact sequence of cohomology (where we have the zero on the left thanks to our assumption that $\chi$ is non-trivial)

$$
0=H^{0}(\mathbb{Q}, T) \longrightarrow H^{1}(\mathbb{Q}, T \otimes \Lambda) \stackrel{\gamma-1}{\longrightarrow} H^{1}(\mathbb{Q}, T \otimes \Lambda) \stackrel{\mathbb{N}}{\longrightarrow} H^{1}(\mathbb{Q}, T) .
$$

It follows from Proposition II.1.1 in [Col98] that we may identify $H^{1}(\mathbb{Q}, T \otimes \Lambda)$ with $\lim _{n} H^{1}\left(\mathbb{Q}_{n}, T\right)$, and thus view the wild cyclotomic unit $\xi$ as an element of $H^{1}(\overleftarrow{Q}, T \otimes \Lambda)$. The image of $\xi$ under the map $\mathbb{N}$ of (3.1) is $\xi_{L}^{\chi}=1$, hence the exact sequence (3.1) shows: 
Proposition 3.4. There exists a unique

$$
\left\{z_{n}^{\chi}\right\}=z_{\infty}^{\chi} \in H^{1}(\mathbb{Q}, T \otimes \Lambda)=\overleftarrow{\lim }_{n} H^{1}\left(\mathbb{Q}_{n}, T\right)
$$

such that

$$
\frac{\gamma-1}{\log _{p} \rho_{\text {cyc }}(\gamma)} \times z_{\infty}^{\chi}=\xi .
$$

Remark 3.5. Just as we did above, one could have obtained an element $z_{\infty} \in$ $\lim _{n} H^{1}\left(L_{n}, \mathbb{Z}_{p}(1)\right)$ such that $\frac{\gamma-1}{\log _{p} \rho_{\text {cyc }}(\gamma)} \times z_{\infty}=\xi_{\infty}:=\left\{\xi_{n}\right\}$. Then, $\chi$-part of this element would be our $z_{\infty}^{\chi}$ and $\xi_{\infty}^{\chi}=\xi$, respectively. Although we only need to analyze the $\chi$-parts $z_{\infty}^{\chi}$ and $\xi=\xi_{\infty}^{\chi}$ of these elements for our purposes, it may be worthwhile to keep this in mind for a comparison with the treatment of [Sol92] and $\S 9.3$ of [BG03].

3.2. Wild cyclotomic $p$-units. In this section we quickly review Solomon's [So192] construction of cyclotomic $p$-units and relate these $p$-units to $z_{\infty}^{\chi}$ defined above.

Solomon's construction ${ }^{3}$ starts with the observation that there exists (thanks to Hilbert 90) a unique $\beta_{n}^{\chi} \in L_{n}^{\times, \chi} / L^{\times, \chi}$ such that

$$
\frac{\gamma-1}{\log _{p} \rho_{\text {cyc }}(\gamma)} \times \beta_{n}^{\chi}=\xi_{n}^{\chi} .
$$

Thus, from our definition of $z_{\infty}^{\chi}=\left\{z_{n}^{\chi}\right\}$ it follows that

$$
\beta_{n}^{\chi}=z_{n}^{\chi} \text { inside } L_{n}^{\times, \chi} / L^{\times, \chi} .
$$

Applying $\mathbf{N}_{L_{n} / L}$ on both sides of this equality we see that

$$
\kappa_{n}^{\chi}:=\mathbf{N}_{L_{n} / L} \beta_{n}^{\chi} \equiv \mathbf{N}_{L_{n} / L} z_{n}^{\chi}=z_{0}^{\chi} \bmod p^{n} .
$$

Solomon proves (and (3.2) above shows as well) that

$$
\kappa_{n^{\prime}}^{\chi} \equiv \kappa_{n}^{\chi} \bmod p^{n}, \text { for } n^{\prime} \geq n
$$

and he defines

$$
\kappa^{\chi}:=\lim _{\longleftarrow} \kappa_{n}^{\chi} \in L^{\times, \chi} .
$$

This is what he calls the cyclotomic p-unit. By (3.2), we clearly have $\kappa^{\chi}=z_{0}^{\chi}$.

\footnotetext{
${ }^{3}$ The attentive reader will notice that Solomon's construction is carried out without taking $\chi$-parts. However his arguments apply on the $\chi$-parts verbatim. In fact, it is easy to see that the $p$-unit $\kappa^{\chi}$ constructed below is simply the $\chi$-part of the $p$-unit $\kappa$ which Solomon constructs in $\S 2$ of [Sol92].
} 
Definition 3.6. The element $z_{0}^{\chi}$ is called the cyclotomic $p$-unit and the collection

$$
z_{\infty}^{\chi} \in \underset{\leftarrow}{\lim } H^{1}\left(\mathbb{Q}_{n, p}, T\right)=\underset{\lim _{n}}{L_{n}^{\times, \chi}}
$$

is called the collection of wild cyclotomic p-units.

Remark 3.7. By [Sol94] that $\left\{c_{1}^{\chi}, z_{0}^{\chi}\right\}$ is an ordered $\mathfrak{F}$-basis for $\tilde{H}_{f}^{1}(\mathbb{Q}, T) \otimes \mathfrak{F}$.

3.3. Local Tate duality. In this section we give a review of well-known results from local duality which we will need later in $\S 4$. For each $n \geq 0$, we have the local Tate pairing

$$
H^{1}\left(\mathbb{Q}_{n, p}, T\right) \times H^{1}\left(\mathbb{Q}_{n, p}, T^{*}\right) \longrightarrow \mathcal{O},
$$

induced from cup-product pairing composed with the invariant isomorphism, for more details see $\$ 5.1-\$ 5.2$ of [Nek06]. This induces a map

$$
H^{1}\left(\mathbb{Q}_{n, p}, T\right) \stackrel{\tau_{n}}{\longrightarrow} \operatorname{Hom}\left(H^{1}\left(\mathbb{Q}_{n, p}, T^{*}\right), \mathcal{O}\right)
$$

thus, in the limit a map (using Proposition II.1.1 in [Col98] once again)

$$
\left.H^{1}\left(\mathbb{Q}_{p}, T \otimes \Lambda\right) \stackrel{\tau_{\infty}}{\longrightarrow} \operatorname{Hom} \underset{n}{\lim } H^{1}\left(\mathbb{Q}_{n, p}, T^{*}\right), \mathcal{O}\right) .
$$

Definition 3.8. (i) Let $\mathscr{L}_{\xi}$ be the image of $\xi$ under the compositum $H^{1}(\mathbb{Q}, T \otimes \Lambda) \stackrel{\operatorname{loc}_{p}}{\longrightarrow} H^{1}\left(\mathbb{Q}_{p}, T \otimes \Lambda\right) \stackrel{\tau_{\infty}}{\longrightarrow} \operatorname{Hom}\left(\underset{n}{\lim } H^{1}\left(\mathbb{Q}_{n, p}, T^{*}\right), \mathcal{O}\right)$.

(ii) Let $\mathscr{L}_{\xi}^{\prime}$ be the image of $z_{\infty}^{\chi}$ under the compositum $H^{1}(\mathbb{Q}, T \otimes \Lambda) \stackrel{\tau_{\infty} \circ \operatorname{loc}_{p}}{\longrightarrow} \operatorname{Hom}\left(\underset{n}{\lim } H^{1}\left(\mathbb{Q}_{n, p}, T^{*}\right), \mathcal{O}\right) \longrightarrow \operatorname{Hom}\left(H^{1}\left(\mathbb{Q}_{p}, T^{*}\right), \mathcal{O}\right)$.

Remark 3.9. For $n \geq n^{\prime}$ we have a commutative diagram

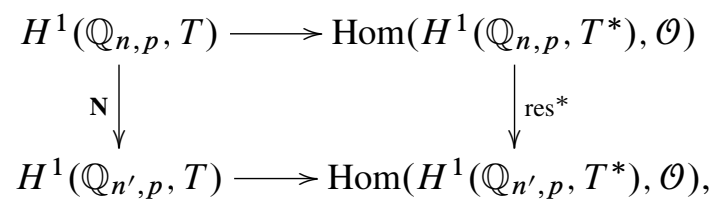

where res* is induced from the restriction map

$$
\text { res: } H^{1}\left(\mathbb{Q}_{n^{\prime}, p}, T^{*}\right) \longrightarrow H^{1}\left(\mathbb{Q}_{n, p}, T^{*}\right) .
$$


We therefore have a commutative diagram

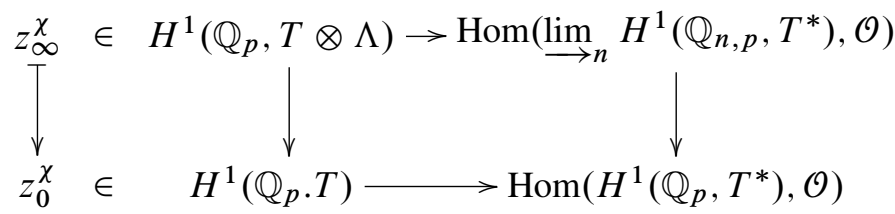

Thus $\mathscr{L}_{\xi}^{\prime}$ is simply the image of $z_{0}^{\chi}$ under the map

$$
\tau_{0}: H^{1}\left(\mathbb{Q}_{p}, T\right) \longrightarrow \operatorname{Hom}\left(H^{1}\left(\mathbb{Q}_{p}, T^{*}\right), \mathcal{O}\right)
$$

\section{Computation of the height pairing}

Throughout $\S 4$, our base field $K$ is $\mathbb{Q}$ and $\chi$ is an even, non-trivial Dirichlet character whose order is prime to $p$, and which has the property that $\chi(p)=1$. In this section we calculate the height pairing on the cyclotomic unit $c_{1}^{\chi}$. Note that, in view of Remark 3.7, Proposition 2.7 and the fact that $z_{0}^{\chi} \in \widetilde{H}_{f}^{1}(\mathbb{Q}, T)$ is a universal norm (by its definition), this gives the only non-trivial output of the machinery we described in $\$ 2$ we could hope for.

For $\psi=\chi^{ \pm 1}$, we write as usual $\mathcal{O}(\psi)$ for the free $\mathcal{O}$-module of rank one, on which $G_{\mathbb{Q}}$ acts via $\psi$. Define $e_{\psi}:=\sum_{\delta \in \Delta} \psi^{-1}(\delta) \delta$ as the idempotent of $\mathcal{O}[\Delta]$ associated to $\psi$. We identify the module $\mathcal{O}(\psi)$ with $\left(\bigoplus_{v \mid p} \mathcal{O} \cdot v\right)^{\psi}$ (therefore we regard $g_{\psi}:=e_{\psi} v_{0}$ as a generator of $\mathcal{O}(\psi)$, where we recall that $v_{0}$ is the place of $L$ we fixed in $\$ 1.1$ via choosing an embedding $\left.\iota_{p}: \overline{\mathbb{Q}} \hookrightarrow \overline{\mathbb{Q}}_{p}\right)$ and we define

$$
\operatorname{pr}_{\psi}:\left(\bigoplus_{v \mid p} \mathcal{O} \cdot v\right)^{\psi} \stackrel{\sim}{\longrightarrow} \mathcal{O}
$$

by setting $\mathfrak{p r}_{\psi}: \mathfrak{g}_{\psi} \mapsto 1$. In other words, $\mathfrak{p r}_{\psi}$ is the map induced from projection onto the $v_{0}$-coordinate. For each place $v$ of $L$ lying above $p$, write $\sigma_{v}: L \hookrightarrow L_{v}=\mathbb{Q}_{p}$ for the induced embedding.

Let $\rho_{\chi}$ denote the compositum

$$
\tilde{H}_{f}^{1}\left(\mathbb{Q}, \mathcal{O}(1) \otimes \chi^{-1}\right) \stackrel{\beta_{\chi}^{1}}{\longrightarrow} \tilde{H}_{f}^{2}\left(\mathbb{Q}, \mathcal{O}(1) \otimes \chi^{-1}\right) \stackrel{\iota}{\longrightarrow} H^{2}\left(\mathbb{Q}, \mathcal{O}(1) \otimes \chi^{-1}\right)
$$


and $\beta_{\chi}$ the compositum

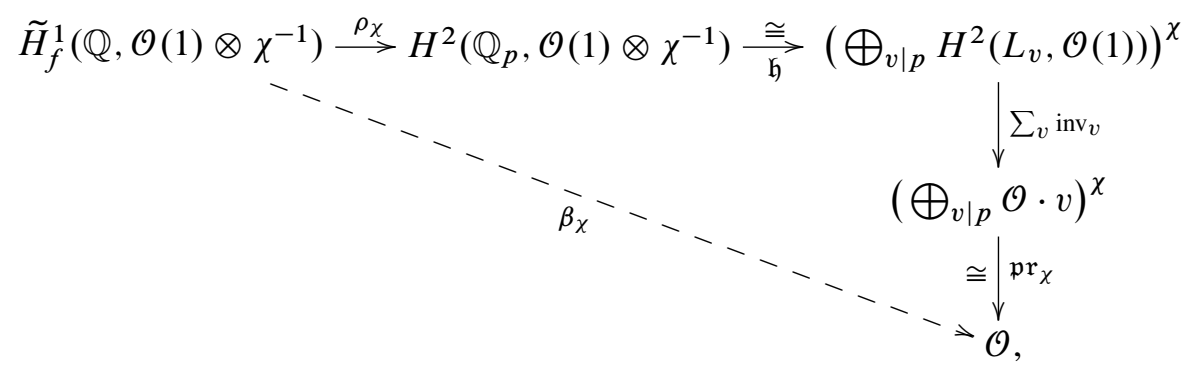

where the map $\beta_{\chi}^{1}$ in the first diagram is the Bockstein morphism applied on the first cohomology; $\iota$ comes from Proposition 2.3; the isomorphism $\mathfrak{h}$ in the second diagram from the Hochschild-Serre spectral sequence. Let $\log _{p}: \widehat{\mathbb{Q}}_{p}^{\times} \rightarrow \mathbb{Z}_{p}$ be the $p$-adic logarithm extended to the $p$-adic completion $\widehat{\mathbb{Q}_{p}^{\times}}$of $\mathbb{Q}_{p}^{\times}$by setting $\log _{p}(p)=0$. We extend $\log _{p}$ by linearity to define an $\mathcal{O}$-module homomorphism

$$
\log _{p}: \mathcal{O} \otimes_{\mathbb{Z}_{p}} \widehat{\mathbb{Q}_{p}^{\times}} \longrightarrow \mathcal{O}
$$

Proposition 4.1. $\beta_{\chi}\left(c_{1}^{\chi}\right)=\log _{p}\left(\iota_{p}\left(c_{1}^{\chi}\right)\right)=v_{0}\left(z_{0}^{\chi}\right) \in \mathcal{O}$.

Proof. The second equality is the main calculation of [Sol92], hence it suffices to check the first claimed equality. This assertion is essentially Proposition 9.3 (ii) in [BG03]. In fact, the statement of loc.cit. is that

$$
\beta_{\chi}\left(c_{1}^{\chi}\right)=\mathfrak{p r}_{\chi}\left(e_{\chi} \sum_{v \mid p} \log _{p}\left(\sigma_{v}\left(c_{1}\right)\right) \cdot v\right),
$$

where the equality takes place in $\mathcal{O}$. Furthermore, we have the following brute-force calculation:

$$
\begin{aligned}
\mathcal{O}(\chi) \ni e_{\chi} \sum_{v \mid p} \log _{p}\left(\sigma_{v}\left(c_{1}\right)\right) \cdot v & =\sum_{\delta \in \Delta} \chi^{-1}(\delta) \delta \sum_{v \mid p} \log _{p}\left(\sigma_{v}\left(c_{1}\right)\right) \cdot v \\
& =\sum_{\delta \in \Delta} \sum_{v \mid p} \chi^{-1}(\delta) \log _{p}\left(\sigma_{v}\left(c_{1}\right)\right) \cdot v^{\delta} \\
& =\sum_{\delta \in \Delta} \sum_{\omega \mid p} \chi^{-1}(\delta) \log _{p}\left(\sigma_{\omega^{-1}}\left(c_{1}\right)\right) \cdot \omega \\
& =\sum_{\delta \in \Delta} \sum_{\omega \mid p} \chi^{-1}(\delta) \log _{p}\left(\sigma_{\omega}\left(c_{1}^{\delta}\right)\right) \cdot \omega \\
& =\sum_{\omega \mid p} \log _{p}\left(\sigma_{\omega}\left(c_{1}^{\chi}\right)\right) \cdot \omega \in \mathcal{O}(\chi),
\end{aligned}
$$


where $v^{\delta}$ is the place obtained by the action of $\delta \in \Delta$ on the set of places $\{v: v \mid p\}$; and we have the final equality by the $\mathcal{O}$-linearity of $\log _{p}$, and the forth equality thanks to the following commutative diagram:

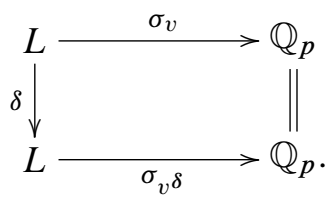

We further have

$$
\begin{aligned}
\sum_{\omega \mid p} \log _{p}\left(\sigma_{\omega}\left(c_{1}^{\chi}\right)\right) \cdot \omega & =\sum_{\delta \in \Delta} \log _{p}\left(\sigma_{v_{0}^{\delta}}\left(c_{1}^{\chi}\right)\right) \cdot v_{0}^{\delta} \\
& =\sum_{\delta \in \Delta} \log _{p}\left(\sigma_{v_{0}}\left(\left(c_{1}^{\chi}\right)^{\delta^{-1}}\right)\right) \cdot v_{0}^{\delta} \\
& =\sum_{\delta \in \Delta} \log _{p}\left(\sigma_{v_{0}}\left(c_{1}^{\chi}\right)^{\chi^{-1}(\delta)}\right) \cdot v_{0}^{\delta} \\
& =\sum_{\delta \in \Delta} \chi^{-1}(\delta) \log _{p}\left(\sigma_{v_{0}}\left(c_{1}^{\chi}\right)\right) \cdot v_{0}^{\delta} \\
& =\log _{p}\left(\sigma_{v_{0}}\left(c_{1}^{\chi}\right)\right) \cdot e_{\chi} v_{0} \in \mathcal{O}(\chi),
\end{aligned}
$$

where the second equality holds thanks to (4.1) and the third because $\left(c_{1}^{\chi}\right)^{\delta^{-1}}=$ $\left(c_{1}^{\chi}\right)^{\chi^{-1}(\delta)}$. Putting all this together (and noting that $\left.\sigma_{v_{0}}\right|_{L}=\left.\iota_{p}\right|_{L}$ by definition), we conclude that

$$
\beta_{\chi}\left(c_{1}^{\chi}\right)=\xi_{\chi}\left(\log _{p}\left(\sigma_{v_{0}}\left(c_{1}^{\chi}\right)\right) \cdot e_{\chi} v_{0}\right)=\log _{p}\left(\iota_{p}\left(c_{1}^{\chi}\right)\right)
$$

as desired.

Remark 4.2. Note that if we replace $v_{0}$ by another place $v_{0}^{\delta}$ of $L$, the value of $\beta_{\chi}\left(c_{1}^{\chi}\right)=$ $\log _{p}\left(\sigma_{v_{0}}\left(c_{1}^{\chi}\right)\right)$ changes by $\chi^{-1}(\delta): \log _{p}\left(\sigma_{v_{0}^{\delta}}\left(c_{1}^{\chi}\right)\right)=\chi^{-1}(\delta) \log _{p}\left(\sigma_{v_{0}}\left(c_{1}^{\chi}\right)\right)$.

We are now ready to complete the computation of Nekovár's height pairing $\left\langle c_{1}^{\chi}, \alpha\right\rangle_{\text {Nek }}$ for $\alpha \in \widetilde{H}_{f}^{1}\left(\mathbb{Q}, T^{*}\right)$ and $c_{1}^{\chi}$ as above. We have the following identifications:

$$
\tilde{H}_{f}^{1}\left(\mathbb{Q}, T^{*}\right) \stackrel{\sim}{\longrightarrow} H^{0}\left(\mathbb{Q}_{p}, \mathcal{O}(\chi)\right) \stackrel{\sim}{\longrightarrow}\left(\bigoplus_{v \mid p} \mathcal{O} \cdot v\right)^{\chi^{-1} \stackrel{\mathfrak{p r}_{\chi}-1}{\longrightarrow}} \stackrel{\longrightarrow}{\longrightarrow} .
$$

Let $\alpha\left(v_{0}\right)$ denote the image of $\alpha$ under the compositum of the maps (4.2).

Remark 4.3. Note that since $\mathfrak{p r}_{\chi^{-1}}$ depends on the choice of $v_{0}$, so does $\alpha\left(v_{0}\right) \in \mathcal{O}$. Write $\mathfrak{p r}_{\chi^{-1}}=\mathfrak{p r}_{\chi^{-1}}\left(v_{0}\right)$ only in this remark to remind us the dependence on $v_{0}$. One then has $\mathfrak{p r}_{\chi^{-1}}\left(v_{0}^{\delta}\right)=\chi(\delta) \mathfrak{p r}_{\chi^{-1}}\left(v_{0}\right)$ and in turn $\alpha\left(v_{0}^{\delta}\right)=\chi(\delta) \alpha\left(v_{0}\right)$. 
Lemma 4.4. Suppose $x \in H^{0}\left(\mathbb{Q}_{p}, \mathcal{O}\right)=\mathcal{O}$ and $y \in H^{2}\left(\mathbb{Q}_{p}, \mathcal{O}(1)\right)$. Then

(i) $x \cup y=x \cdot y \in H^{2}\left(\mathbb{Q}_{p}, \mathcal{O}(1)\right)$,

(ii) $\langle x, y\rangle_{\text {Tate }}=x \cdot \operatorname{inv}_{p}(y) \in \mathcal{O}$, where $\langle\text {, }\rangle_{\text {Tate }}$ is the local Tate pairing.

Proof. Clear.

Lemma 4.4 may be used to check the following:

Lemma 4.5. The following diagram commutes:

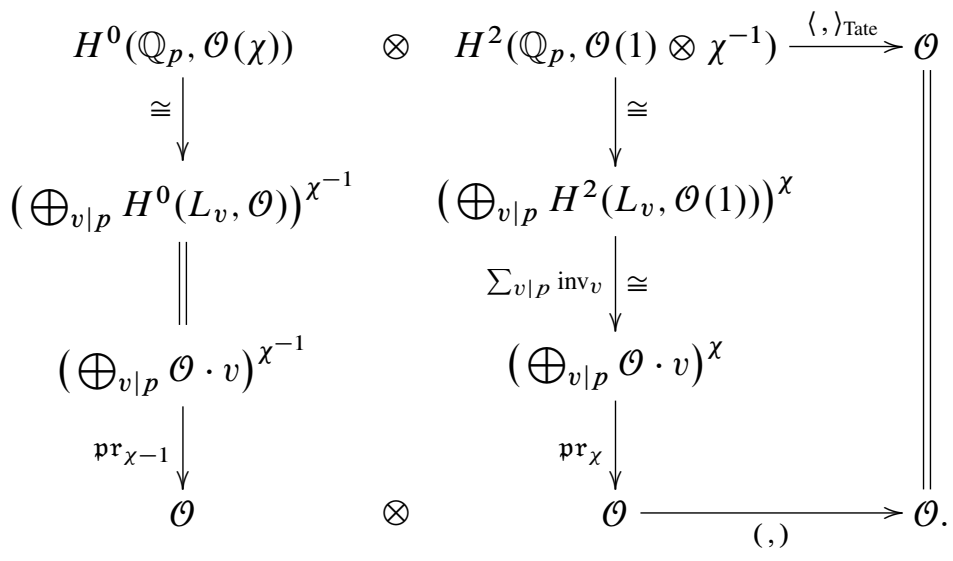

Here, $(a, b):=a b \in \mathcal{O}$ for $a, b \in \mathcal{O}$, and the vertical isomorphisms between first two rows come from the Hochschild-Serre spectral sequence.

The following proposition is key to our main results.

Proposition 4.6. For an arbitrary $\alpha \in \tilde{H}_{f}^{1}\left(\mathbb{Q}, T^{*}\right)$, we have $\left\langle c_{1}^{\chi}, \alpha\right\rangle_{\mathrm{Nek}}=v_{0}\left(z_{0}^{\chi}\right)$. $\alpha\left(v_{0}\right)$.

Remark 4.7. Both $v_{0}\left(z_{0}^{\chi}\right)$ and $\alpha\left(v_{0}\right)$ depend on the choice of $v_{0}$, yet $v_{0}\left(z_{0}^{\chi}\right) \cdot \alpha\left(v_{0}\right)$ is independent of $v_{0}$ thanks to Remarks 4.2 and 4.3.

Proof. By Proposition 2.12

$$
\left\langle c_{1}^{\chi}, \alpha\right\rangle_{\mathrm{Nek}}=\left\langle\beta_{\chi}^{1}\left(c_{1}^{\chi}\right), \alpha\right\rangle_{\mathrm{PT}}
$$

where

$$
\langle,\rangle_{\mathrm{PT}}: \tilde{H}_{f}^{2}(\mathbb{Q}, T) \otimes \tilde{H}_{f}^{1}\left(\mathbb{Q}, T^{*}\right) \longrightarrow \mathcal{O}
$$


denotes the global pairing from [Nek06], §6.3. The definition of this global pairing (along with the fact that $H^{2}\left(\mathbb{Q}_{\ell}, T\right)=0$ for every $\left.\ell \mid f_{\chi}\right)$ shows that the following diagram commutes:

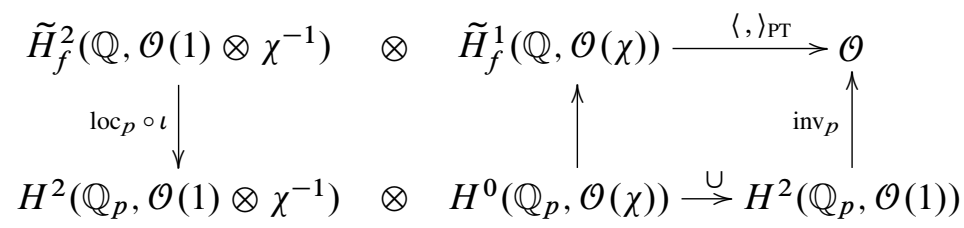

We explain the arrows in (4.3): The arrow on the left is the canonical map (coming from Proposition 2.3)

$$
\iota: \tilde{H}_{f}^{2}\left(\mathbb{Q}, \mathcal{O}(1) \otimes \chi^{-1}\right) \longrightarrow H^{2}\left(\mathbb{Q}, \mathcal{O}(1) \otimes \chi^{-1}\right)
$$

followed by the restriction map $\operatorname{loc}_{p}$. The extended Selmer group $\widetilde{H}_{f}^{1}(\mathbb{Q}, \mathcal{O}(\chi))$ may be canonically identified by $H^{0}\left(\mathbb{Q}_{p}, \mathcal{O}(\chi)\right)$ (see $\left.\S 2.2\right)$, this is how we obtain the vertical arrow in the center.

The commutative diagram (4.3) gives $\left\langle c_{1}^{\chi}, \alpha\right\rangle_{\text {Nek }}=\left\langle\rho_{\chi}\left(c_{1}^{\chi}\right), \alpha\right\rangle_{\text {Tate }}$, where $\rho_{\chi}$ is defined as in the beginning of $\S 4$. Furthermore, by Lemma 4.5

$$
\left\langle\rho_{\chi}\left(c_{1}^{\chi}\right), \alpha\right\rangle_{\text {Tate }}=\left(\beta_{\chi}\left(c_{1}^{\chi}\right), \alpha\left(v_{0}\right)\right)=v_{0}\left(z_{0}^{\chi}\right) \cdot \alpha\left(v_{0}\right),
$$

where $(a, b):=a \cdot b$ for $a, b \in \mathcal{O}$ as in Lemma 4.5 , and the final equality is Proposition 4.1. The proof is now complete.

\section{Rubin's formula}

Throughout $\S 4$, our base field $K$ is $\mathbb{Q}$ and $\chi$ is an even, non-trivial Dirichlet character whose order is prime to $p$, and which has the property that $\chi(p)=1$. In this section we complete our main computation, using the calculations carried out in $\S 4$. Starting with $\alpha \in \widetilde{H}_{f}^{1}(\mathbb{Q}, \mathcal{O}(\chi))$ as above, we first wish to define an element $\phi_{\alpha}$ :

$$
\begin{aligned}
\phi_{\alpha} \in H^{1}\left(\mathbb{Q}_{p}, \mathcal{O}(\chi)\right) & =\left(\bigoplus_{v \mid p} H^{1}\left(L_{v}, \mathcal{O}\right)\right)^{\chi^{-1}} \underset{\mathfrak{p r}_{\chi^{-1}}}{\sim} H^{1}\left(L_{v_{0}}, \mathcal{O}\right) \\
& =\operatorname{Hom}\left(G_{v_{0}}, \mathcal{O}\right) .
\end{aligned}
$$

Here we recall that $G_{v}=\operatorname{Gal}\left(\overline{\mathbb{Q}}_{p} / L_{v}\right)$ and $\mathfrak{p r}_{\chi^{-1}}$ is the projection onto the $v_{0^{-}}$ coordinate as in $\$ 4$. In the equalities above, we are again using an identification coming from Hochschild-Serre spectral sequence, along with the fact that $H^{1}\left(L_{v}, \mathcal{O}\right)=$ $\operatorname{Hom}\left(G_{v}, \mathcal{O}\right)$. Note also that $\operatorname{Hom}\left(G_{v}, \mathcal{O}\right)$ is the group of continuous homomorphisms and we have

$$
\operatorname{Hom}\left(G_{v}, \mathcal{O}\right)=\operatorname{Hom}\left(G_{v}^{\mathrm{ab}}, \mathcal{O}\right)=\operatorname{Hom}\left(G_{v}^{\mathrm{ab}, p}, \mathcal{O}\right)=\operatorname{Hom}_{\mathcal{O}}\left(\mathcal{O} \otimes_{\mathbb{Z}_{p}} G_{v}^{\mathrm{ab}, p}, \mathcal{O}\right),
$$


where $G_{v}^{\mathrm{ab}}$ for the abelianization of the group $G_{v}$ and $G_{v}^{\mathrm{ab}, p}$ is its pro- $p$ part.

We write $\phi_{\alpha}^{v_{0}} \in \operatorname{Hom}\left(G_{v_{0}}, \mathcal{O}\right)$ for the image of $\phi_{\alpha}$ under the compositum (5.1) (which we henceforth call $\mathfrak{r}_{\chi}$ ). Defining $\phi_{\alpha}^{v_{0}}$ as the unramified homomorphism given by

$$
\phi_{\alpha}^{v_{0}}: G_{v_{0}} \longrightarrow \mathcal{O}, \quad \mathrm{Fr}_{v_{0}} \longmapsto \alpha\left(v_{0}\right)
$$

where $\mathrm{Fr}_{v_{0}}$ denotes an arithmetic Frobenius at $v_{0}$, we also define $\phi_{\alpha} \in H^{1}\left(\mathbb{Q}_{p}, \mathcal{O}(\chi)\right)$ using the identification $r_{\chi}$. Below, we normalize the local reciprocity isomorphism (and the local invariant map) by letting uniformizers correspond to arithmetic Frobenius elements.

Let $\xi=\xi_{\infty}^{\chi}=\left\{\xi_{n}^{\chi}\right\} \in H^{1}(\mathbb{Q}, T \otimes \Lambda)$ be the collection of wild cyclotomic units, as in $\S 3$. Recall the definition of the element $\mathscr{L}_{\xi}^{\prime} \in H^{1}\left(\mathbb{Q}_{p}, T\right)$ from $\S 3.3$ which we regard as an element of $\operatorname{Hom}\left(H^{1}\left(\mathbb{Q}_{p}, T^{*}\right), \mathcal{O}\right)$ via local duality. Recall also the tame cyclotomic unit $c_{1}^{\chi} \in H^{1}(\mathbb{Q}, T)$.

Theorem 5.1. $\left\langle c_{1}^{\chi}, \alpha\right\rangle_{\mathrm{Nek}}=\mathscr{L}_{\xi}^{\prime}\left(\phi_{\alpha}\right)$.

Proof. Let $z_{0}^{\chi}$ be Solomon's cyclotomic $p$-unit as above. It follows from the discussion in $\$ 3.3$ that

$$
\mathscr{L}_{\xi}^{\prime}\left(\phi_{\alpha}\right)=\left\langle z_{0}^{\chi}, \phi_{\alpha}\right\rangle_{\text {Tate }} .
$$

The computation of the right hand side of Theorem 5.1 is thus reduced to local class field theory.

Let $r_{\chi^{-1}}$ denote the following compositum:

$$
\begin{aligned}
H^{1}\left(\mathbb{Q}_{p}, \mathcal{O}(1) \otimes \chi^{-1}\right) & =\left(\bigoplus_{v \mid p} H^{1}\left(L_{v}, \mathcal{O}(1)\right)\right)^{\chi} \underset{\xi_{\chi}}{\stackrel{\sim}{\longrightarrow}} H^{1}\left(L_{v_{0}}, \mathcal{O}(1)\right) \\
& =\widehat{L_{v_{0}}^{\times}} \otimes_{\mathbb{Z}_{p}} \mathcal{O}
\end{aligned}
$$

where $\xi_{\chi}$ is the projection onto the $v_{0}$-coordinate as above, and $\widehat{L_{v}^{\times}}$stands for the $p$-adic completion of the multiplicative group $L_{v}^{\times}$. We note that $\mathfrak{r}_{\chi^{-1}}\left(\operatorname{loc}_{p}\left(z_{0}^{\chi}\right)\right)=$ $\iota_{p}\left(z_{0}^{\chi}\right)$, with $\iota_{p}: L \hookrightarrow L_{v_{0}}$ is as in the introduction and

$$
\operatorname{loc}_{p}: H^{1}(\mathbb{Q}, T) \rightarrow H^{1}\left(\mathbb{Q}_{p}, T\right)
$$

is the canonical restriction map, as usual. We then have a commutative diagram

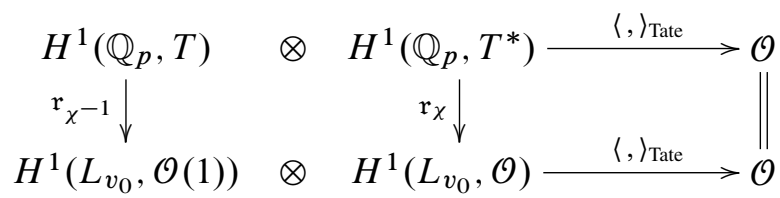


which translates to

$$
\left\langle z_{0}^{\chi}, \phi_{\alpha}\right\rangle_{\mathrm{Tate}}=\left\langle\iota_{p}\left(z_{0}^{\chi}\right), \phi_{\alpha}^{v_{0}}\right\rangle_{\mathrm{Tate}} .
$$

Let

$$
\mathfrak{a}_{v}: H^{1}\left(L_{v}, \mathbb{Z}_{p}(1)\right)=\widehat{L_{v}^{\times}} \longrightarrow G_{v}^{\mathrm{ab}, p}
$$

denote the local reciprocity map. Let further

$$
\mathrm{a}_{v}^{(\mathrm{ur})}: \widehat{L_{v}^{\times}} \longrightarrow \operatorname{Gal}\left(L_{v}^{\mathrm{ur}} / L_{v}\right)
$$

denote the projection of $a_{v}$ to the Galois group of the maximal unramified extension of $L_{v}$. We also write $a_{v}$ (resp., $\mathfrak{a}_{v}^{(\text {ur) }}$ ) for the induced map $\mathcal{O} \otimes_{\mathbb{Z}_{p}} \widehat{L_{v}^{\times}} \rightarrow \mathcal{O} \otimes_{\mathbb{Z}_{p}} G_{v}^{\text {ab, } p}$ (resp., for the map $\mathcal{O} \otimes_{\mathbb{Z}_{p}} \widehat{L_{v}^{\times}} \rightarrow \mathcal{O} \otimes_{\mathbb{Z}_{p}} \operatorname{Gal}\left(L_{v}^{\mathrm{ur}} / L_{v}\right)$ ).

By the very definition of the local Tate pairing,

$$
\left\langle\iota_{p}\left(z_{0}^{\chi}\right), \phi_{\alpha}^{v_{0}}\right\rangle_{\text {Tate }}=\phi_{\alpha}^{v_{0}}\left(a_{v_{0}}\left(\iota_{p}\left(z_{0}^{\chi}\right)\right)\right)=\phi_{\alpha}^{v_{0}}\left(\mathfrak{a}_{v_{0}}^{(\mathrm{ur})}\left(\iota_{p}\left(z_{0}^{\chi}\right)\right)\right)
$$

where we have the second equality because $\phi_{\alpha}^{v_{0}}$ is unramified by construction. Write

$$
\iota_{p}\left(z_{0}^{\chi}\right)=\varpi_{v_{0}}^{v_{0}\left(z_{0}^{\chi}\right)} \cdot u \in \mathcal{O} \otimes_{\mathbb{Z}_{p}} \widehat{L_{v_{0}}^{\widehat{x}}}=\mathcal{O} \otimes_{\mathbb{Z}_{p}}\left(\varpi_{v_{0}}^{\mathbb{Z}_{p}} \oplus \widehat{\mathcal{O}_{L_{v_{0}}}^{\times}}\right),
$$

where $\varpi_{v_{0}}$ is a uniformizer of $L_{v_{0}}$ and $u \in \mathcal{O} \otimes_{\mathbb{Z}_{p}} \widehat{\mathcal{O}_{L_{v_{0}}}^{\times}}$is a unit at $v_{0}$. Then $a_{v_{0}}^{(\text {ur) }}\left(\iota_{p}\left(z_{0}^{\chi}\right)\right)=\operatorname{Fr}_{v_{0}}^{v_{0}\left(z_{0}^{\chi}\right)}$ since $a_{v_{0}}(u) \in d_{v} \subset G_{v}$, the inertia subgroup at $v$. Thus

$$
\left\langle\iota_{p}\left(z_{0}^{\chi}\right), \phi_{\alpha}^{v_{0}}\right\rangle_{\text {Tate }}=\phi_{\alpha}^{v_{0}}\left(\operatorname{Fr}_{v_{0}}^{v_{0}\left(z_{0}^{\chi}\right)}\right)=v_{0}\left(z_{0}^{\chi}\right) \cdot \phi_{\alpha}^{v_{0}}\left(\operatorname{Fr}_{v_{0}}\right)
$$

and this equals, by the definition of $\phi_{\alpha}^{v_{0}}$, to $v_{0}\left(z_{0}^{\chi}\right) \cdot \alpha\left(v_{0}\right)$, which equals, by Proposition 4.6 to $\left\langle z_{0}^{\chi}, \alpha\right\rangle_{\mathrm{Nek}}$ and finally, by (5.2) and (5.4) to $\mathscr{L}_{\xi}^{\prime}\left(\phi_{\alpha}\right)$. This completes the proof.

Next, we relate the right hand side of the statement of Theorem 5.1 to a special value of a $p$-adic $L$-function (that we call $L_{\xi, \Phi}$ ) which we construct below.

Let $\Phi_{\infty}$ denote the cyclotomic $\mathbb{Z}_{p}$-extension of $\mathbb{Q}_{p}:=\Phi_{0}$, and let $\Phi_{n}$ denote the unique sub-extension of $\Phi_{\infty} / \mathbb{Q}_{p}$ of degree $p^{n}$. Recall that $T^{*}=\operatorname{Hom}(T, \mathcal{O}(1)) \cong$ $\mathcal{O}(\chi)$. We set

$$
H_{\infty}^{1}\left(\mathbb{Q}_{p}, T^{*}\right)={\underset{\lim }{n}}_{n} H^{1}\left(\Phi_{n}, T^{*}\right)
$$

where the inverse limit is taken with respect to norm maps. We may identify $\operatorname{Gal}\left(\Phi_{\infty} / \mathbb{Q}_{p}\right)$ naturally by $\Gamma=\operatorname{Gal}\left(\mathbb{Q}_{\infty} / \mathbb{Q}\right)$. Let $\gamma$ be a topological generator for $\Gamma$ and let $\Lambda=\mathcal{O} \llbracket \Gamma \rrbracket$ as usual.

Lemma 5.2. The natural map $H_{\infty}^{1}\left(\mathbb{Q}_{p}, T^{*}\right) \rightarrow H^{1}\left(\mathbb{Q}_{p}, T^{*}\right)$ is surjective. 
Proof. By [Co198], Proposition II.1.1, we have $H_{\infty}^{1}\left(\mathbb{Q}_{p}, T^{*}\right) \cong H^{1}\left(\mathbb{Q}_{p}, T^{*} \otimes \Lambda\right)$ and the map above is simply the reduction map modulo $\gamma-1$. Hence, the cokernel of this map is $H^{2}\left(\mathbb{Q}_{p}, T^{*} \otimes \Lambda\right)[\gamma-1]$, the $\gamma-1$ torsion of $H^{2}\left(\mathbb{Q}_{p}, T^{*} \otimes \Lambda\right)$. Since the cohomological dimension of $\operatorname{Gal}\left(\overline{\mathbb{Q}}_{p} / \mathbb{Q}_{p}\right)$ is 2 , it follows that

$$
H^{2}\left(\mathbb{Q}_{p}, T^{*} \otimes \Lambda\right) /(\gamma-1) \cong H^{2}\left(\mathbb{Q}_{p}, T^{*} \otimes \Lambda /(\gamma-1)\right)=H^{2}\left(\mathbb{Q}_{p}, T^{*}\right),
$$

which is trivial (by local duality). Thus we have an exact sequence

$$
\begin{aligned}
0 \longrightarrow & H^{2}\left(\mathbb{Q}_{p}, T^{*} \otimes \Lambda\right)[\gamma-1] \longrightarrow H^{2}\left(\mathbb{Q}_{p}, T^{*} \otimes \Lambda\right) \\
& \stackrel{\gamma-1}{\longrightarrow} H^{2}\left(\mathbb{Q}_{p}, T^{*} \otimes \Lambda\right) \longrightarrow 0 .
\end{aligned}
$$

It is known that $H^{2}\left(\mathbb{Q}_{p}, T^{*} \otimes \Lambda\right)$ is an $\mathcal{O}$-module of finite type (cf. Proposition 3.2.1 in [PR94]), thus it follows from Theorem 2.4 in [Mat89] that $H^{2}\left(\mathbb{Q}_{p}, T^{*} \otimes \Lambda\right)[\gamma-1]=$ 0 as well, hence the lemma is proved.

By Lemma 5.2, it is possible to choose $\Phi=\left\{\phi_{\alpha}^{(n)}\right\}_{n \geq 0} \in H_{\infty}^{1}\left(\mathbb{Q}_{p}, T^{*}\right)$ such that $\phi_{\alpha}^{(0)}=\phi_{\alpha}$.

Definition 5.3. Attached to $\xi$ and $\Phi$, define an $\mathcal{O}$-valued measure $\mu_{\xi, \Phi}$ on $\Gamma$ as follows: For $\tau \in \Gamma$, set

$$
\mu_{\xi, \Phi}\left(\tau \Gamma^{p^{n}}\right)=\mathscr{L}_{\xi}\left(\tau \phi_{\alpha}^{(n)}\right)
$$

The fact that $\mu_{\xi, \Phi}$ is a distribution follows from the fact that the collection $\left\{\Phi_{\alpha}^{(n)}\right\}_{n}$ is norm-compatible.

We define the " $p$-adic $L$-function" associated to $\xi$ and $\Phi$ by setting

$$
L_{\xi, \Phi}(\eta)=\int_{\Gamma} \eta d \mu_{\xi, \Phi}
$$

for each character $\eta: \Gamma \rightarrow \mathbb{Z}_{p}^{\times}$. Let 1 be the trivial character, and $\rho_{\text {cyc }}: \Gamma \rightarrow 1+p \mathbb{Z}_{p}$ be the cyclotomic character. We define the derivative at the trivial character $\mathbf{1}$ as

$$
L_{\xi, \Phi}^{\prime}(\mathbf{1}):=\left.\frac{d}{d s} L_{\xi, \Phi}\left(\rho_{\text {cyc }}^{s}\right)\right|_{s=0} .
$$

We also define $\mathfrak{P}_{\xi, \Phi} \in \Lambda$ to be the power series associated with the measure $\mu_{\xi, \Phi}$.

Remark 5.4. Define

$$
P_{n}\left(\mu_{\xi, \Phi}\right):=\sum_{\tau \in \Gamma / \Gamma p^{n}} \mu_{\xi, \Phi}\left(\tau \Gamma^{p^{n}}\right) \cdot \tau \in \mathcal{O}\left[\Gamma / \Gamma^{p^{n}}\right],
$$

so that $\mathfrak{P}_{\xi, \Phi}=\lim _{n} P_{n}\left(\mu_{\xi, \Phi}\right) \in \mathcal{O}[[\Gamma]]$. For the powers $\rho_{\text {cyc }}^{s}: \Gamma \rightarrow 1+p \mathbb{Z}_{p}$ of the cyclotomic character, observe that

$$
\rho_{\text {cyc }}^{s}\left(\mathfrak{P}_{\xi, \Phi}\right)=\lim _{n \rightarrow \infty} \sum_{\tau \in \Gamma / \Gamma p^{n}} \mu_{\xi, \Phi}\left(\tau \Gamma^{p^{n}}\right) \cdot \rho_{\text {cyc }}^{s}(\tilde{\tau}) .
$$


Here, $\tilde{\tau} \in \Gamma$ stands for an arbitrary lift of $\tau \in \Gamma / \Gamma^{p^{n}}$, and it is not hard to see that the limit above does not depend on the choice of these lifts although each sum does depend on this choice. We therefore see that $\rho_{\text {cyc }}^{s}\left(\mathfrak{\beta}_{\xi, \Phi}\right)=L_{\xi, \Phi}\left(\rho_{\text {cyc }}^{s}\right)$, which in turn implies that

$$
\left.\frac{d}{d s} \rho_{\mathrm{cyc}}^{s}\left(\mathfrak{P}_{\xi, \Phi}\right)\right|_{s=0}=L_{\xi, \Phi}^{\prime}(\mathbf{1})
$$

Proposition 5.5. $\mathscr{L}_{\xi}^{\prime}\left(\phi_{\alpha}\right)=L_{\xi, \Phi}^{\prime}(1)$

Remark 5.6. Note that the left hand side of the equality in Proposition 5.5 depends only on $\phi_{\alpha}$, not on its lift $\Phi$; whereas the right hand side depends a priori on $\Phi$. Hence Proposition 5.5 shows in particular that $L_{\xi, \Phi}^{\prime}(\mathbf{1})$ does only depend on $\phi_{\alpha}$, and not on the lifting $\Phi$.

Corollary 5.7. $\left\langle c_{1}^{\chi}, \alpha\right\rangle_{\mathrm{Nek}}=L_{\xi, \Phi}^{\prime}(\mathbf{1})$.

The proof of Proposition 5.5 will be completed in a few steps, all of which are essentially borrowed from [Rub94] with minor alterations.

Definition 5.8. Suppose $\mu=\mu^{(0)} \in H^{1}\left(\Phi_{0}, T^{*}\right)$ and $\mu=\left\{\mu^{(n)}\right\} \in \underset{\lim }{\longleftarrow} H^{1}\left(\Phi_{n}, T^{*}\right)$. Define

$$
\operatorname{Der}_{\rho_{c y c}}\left(\mathscr{L}_{\xi}\right)(\mu):=\lim _{n \rightarrow \infty} \sum_{\tau \in \operatorname{Gal}\left(\mathbb{Q}_{n} / \mathbb{Q}\right)} \log _{p}\left(\rho_{\text {cyc }}(\tau)\right) \cdot \mathscr{L}_{\xi}\left(\tau \mu^{(n)}\right) .
$$

As the notation suggests, this definition only depends only on $\mu$, not on the lift $\mu$. This fact will follow from Lemma 5.9 below (where we also prove that the limit above exists).

Lemma 5.9. Suppose $v \in H^{1}\left(\Phi_{n}, T^{*}\right)$ is such that $\mathbf{N}_{\Phi_{n} / \Phi_{0}}(v)=0$. Then

$$
\sum_{\tau \in \operatorname{Gal}\left(\mathbb{Q}_{n} / \mathbb{Q}\right)} \log _{p}\left(\rho_{\text {cyc }}(\tau)\right) \cdot \mathscr{L}_{\xi}(\tau \nu) \equiv 0 \bmod p^{n}
$$

Proof. Fix $n$ and to ease notation, set $\mathscr{L}=\left.\mathscr{L}_{\xi}\right|_{H^{1}\left(\Phi_{n}, T^{*}\right)} \in \operatorname{Hom}\left(H^{1}\left(\Phi_{n}, T^{*}\right), \mathcal{O}\right)$ and $G=\operatorname{Gal}\left(\mathbb{Q}_{n} / \mathbb{Q}\right)$. Write

$$
\delta=\sum_{\tau \in G} \log _{p}\left(\rho_{\text {cyc }}(\tau)\right) \cdot \tau^{-1} \in \mathbb{Z} / p^{n} \mathbb{Z}[G] .
$$

Note that the claim of the lemma is equivalent to showing that

$$
\delta \mathscr{L}(v)=0\left(\text { in } \mathcal{O} / p^{n} \mathcal{O}\right) .
$$


It is easy to see that

$$
\begin{aligned}
(\sigma-1) \delta & =\log _{p}\left(\rho_{\text {cyc }}(\sigma)\right) \sum_{\tau \in G} \tau \\
& =\log _{p}\left(\rho_{\text {cyc }}(\sigma)\right) \cdot \mathbf{N}_{\Phi_{n} / \Phi_{0}}, \quad \text { for all } \sigma \in G,
\end{aligned}
$$

hence it follows that

$$
(\sigma-1) \delta \mathscr{L}=\log _{p}\left(\rho_{\text {cyc }}(\sigma)\right) \cdot \mathbf{N}_{\Phi_{n} / \Phi_{0}} \mathscr{L}=0,
$$

where we have the final equality because $\left.\mathscr{L}\right|_{H^{1}\left(\Phi_{0}, T^{*}\right)}=0$ by Lemma 3.3. This is equivalent to saying that

$$
\delta \mathscr{L} \in \operatorname{Hom}\left(H^{1}\left(\Phi_{n}, T^{*}\right), \mathcal{O} / p^{n} \mathcal{O}\right)^{G} .
$$

Consider the map

$$
\mathbf{N}^{*}: \operatorname{Hom}\left(H^{1}\left(\Phi_{0}, T^{*}\right), \mathcal{O} / p^{n} \mathcal{O}\right) \stackrel{-\circ \mathbf{N}_{\Phi_{n} / \Phi_{0}}}{\longrightarrow} \operatorname{Hom}\left(H^{1}\left(\Phi_{n}, T^{*}\right), \mathcal{O} / p^{n} \mathcal{O}\right)^{G}
$$

Note that both of the sides of above are finite and the map $\mathbf{N}^{*}$ is injective by Lemma 5.2. Claim below proves that there is an isomorphism

$$
\operatorname{Hom}\left(H^{1}\left(\Phi_{n}, T^{*}\right), \mathcal{O} / p^{n} \mathcal{O}\right)^{G} \cong \operatorname{Hom}\left(H^{1}\left(\Phi_{0}, T^{*}\right), \mathcal{O} / p^{n} \mathcal{O}\right)
$$

which in turn implies that $\mathbf{N}^{*}$ is surjective as well:

Claim. $\operatorname{Hom}\left(H^{1}\left(\Phi_{n}, T^{*}\right), \mathcal{O} / p^{n} \mathcal{O}\right)^{G} \cong \operatorname{Hom}\left(H^{1}\left(\Phi_{0}, T^{*}\right), \mathcal{O} / p^{n} \mathcal{O}\right)$.

Proof of the Claim: By slight abuse, we let $\gamma$ denote a generator of $G$. Then, an element $\psi \in \operatorname{Hom}\left(H^{1}\left(\Phi_{n}, T^{*}\right), \mathcal{O} / p^{n} \mathcal{O}\right)$ is fixed by $G$ if and only if

$$
\begin{aligned}
\gamma^{-1} \psi=\psi & \Longleftrightarrow \psi(\gamma x)=\psi(x) \text { for all } x \in H^{1}\left(\Phi_{n}, T^{*}\right) \\
& \Longleftrightarrow \psi((\gamma-1) x)=0 \text { for all } x \in H^{1}\left(\Phi_{n}, T^{*}\right) \\
& \Longleftrightarrow \psi \text { factors through } H^{1}\left(\Phi_{n}, T^{*}\right) /(\gamma-1) \cong H^{1}\left(\Phi_{0}, T^{*}\right) .
\end{aligned}
$$

where the very last isomorphism comes from the proof of Lemma 5.2.

We are now ready to complete the proof of Lemma 5.9. It follows from our conclusion that $\mathbf{N}^{*}$ is surjective that there exists $g \in \operatorname{Hom}\left(H^{1}\left(\Phi_{0}, T^{*}\right), \mathcal{O} / p^{n} \mathcal{O}\right)$ such that $\delta \mathscr{L}=g \circ \mathbf{N}_{\Phi_{n} / \Phi}$, hence

$$
\delta \mathscr{L}(v)=g\left(\mathbf{N}_{\Phi_{n} / \Phi_{0}}(v)\right)=0 \text { in } \mathcal{O} / p^{n} \mathcal{O} .
$$

This is exactly the statement of (5.6). 
Remark 5.10. As in the remark following Lemma 3.1 in [Rub94], one can check that

$$
\operatorname{Der}_{\rho_{c y c}}\left(\mathscr{L}_{\xi}\right)=\mathscr{L}_{\xi}^{\prime}
$$

using the fact that $H^{1}\left(\mathbb{Q}_{p}, T \otimes \Lambda\right)$ has no $(\gamma-1)$-torsion. Here the equality takes place in Hom $\left(H^{1}\left(\Phi_{0}, T^{*}\right), \mathcal{O}\right)$. Note that the term involving the $p$-adic logarithm in loc.cit. does not appear here because we have already normalized $z_{\infty}^{\chi}$ by the factor $\log _{p} \rho_{\text {cyc }}(\gamma)$.

Proof of Proposition 5.5. (Compare with Proposition 7.1 (ii) in [Rub94].) By Remark 5.10,

$$
\begin{aligned}
\mathscr{L}_{\xi}^{\prime}\left(\phi_{\alpha}\right) & =\lim _{n \rightarrow \infty} \sum_{\tau \in \mathrm{Gal}\left(\mathbb{Q}_{n} / \mathbb{Q}\right)} \log _{p} \rho_{\mathrm{cyc}}(\tau) \cdot \mathscr{L}_{\xi}\left(\tau \Phi_{\alpha}^{(n)}\right) \\
& =\lim _{n \rightarrow \infty} \sum_{\tau \in \mathrm{Gal}\left(\mathbb{Q}_{n} / \mathbb{Q}\right)} \log _{p} \rho_{\mathrm{cyc}}(\tau) \mu_{\xi, \Phi}\left(\tau \Gamma^{p^{n}}\right) \\
& =\int_{\Gamma} \log _{p} \rho_{\mathrm{cyc}} \cdot d \mu_{\xi, \Phi .}
\end{aligned}
$$

On the other hand

$$
\frac{d}{d s} \rho_{\mathrm{cyc}}^{s}=\left(\log _{p} \rho_{\mathrm{cyc}}\right) \rho_{\mathrm{cyc}}^{s}
$$

hence

$$
\begin{aligned}
L_{\xi, \Phi}^{\prime}(\mathbf{1})=\left.\frac{d}{d s}\left(\int_{\Gamma} \rho_{\mathrm{cyc}}^{s} \cdot d \mu_{\xi, \Phi}\right)\right|_{s=0} & =\left.\left(\int_{\Gamma}\left(\log _{p} \rho_{\mathrm{cyc}}\right) \rho_{\mathrm{cyc}}^{s} \cdot d \mu_{\xi, \Phi}\right)\right|_{s=0} \\
& =\int_{\Gamma} \log _{p} \rho_{\mathrm{cyc}} \cdot d \mu_{\xi, \Phi} \\
& =\mathscr{L}_{\xi}^{\prime}\left(\phi_{\alpha}\right) .
\end{aligned}
$$

\section{6. $p$-adic $L$-functions and Nekovář's height pairing}

In this section, we obtain a formula for the leading term of an imprimitive KubotaLeopoldt p-adic L-function in terms of Nekovár's height pairing, much in the spirit of a $p$-adic Gross-Zagier formula, using the Rubin-style formula we proved above. This in particular suggests a new interpretation of the classical $p$-adic Kronecker limit formula (cf. [Was82], Theorem 5.18, §2.5 of [dS87]) and the formula of FerreroGreenberg [FG78].

6.1. $p$-adic $\boldsymbol{L}$-functions. In this section, we give an overview of the well-known construction of the Kubota-Leopoldt $p$-adic $L$-function (resp., Katz's two variable $p$-adic $L$-function) using cyclotomic units (resp., elliptic units). 
6.1.1. Cyclotomic units and the Kubota-Leopoldt $\boldsymbol{p}$-adic $\boldsymbol{L}$-function. We denote by $\omega: G_{\mathbb{Q}} \rightarrow\left(\mathbb{Z}_{p}^{\times}\right)_{\text {tors }}$ the Teichmüller character giving the action of $G_{\mathbb{Q}}$ on the $p$-th roots of unity $\mu_{p}$. Fix an embedding $\mathcal{O} \hookrightarrow \overline{\mathbb{Q}}_{p} \hookrightarrow \mathbb{C}$ so that one can identify complex and $p$-adic characters of finite order of $G_{\mathbb{Q}}$. Via this identification, a character $\rho$ of $\Gamma$ of finite order naturally extends to an $\mathcal{O}$-algebra homomorphism $\rho: \Lambda \rightarrow \overline{\mathbb{Q}}_{p}$.

For a character $\rho: G_{\mathbb{Q}} \rightarrow \mathcal{O} \hookrightarrow \mathbb{C}$ of finite order, let $L(s, \rho)$ denote the associated Dirichlet $L$-series .

Definition 6.1. Attached to a non-trivial even Dirichlet character $\psi$ of $G_{\mathbb{Q}}$ whose order is prime to $p$, there is an element $\mathscr{L}_{\psi} \in \Lambda$ such that for every $k \geq 1$ and every character $\rho$ of finite order of $\Gamma$,

$$
\rho_{\text {cyc }}^{k} \rho\left(\mathscr{L}_{\psi}\right)=\left(1-\omega^{-k} \rho \psi(p) p^{k-1}\right) L\left(1-k, \omega^{-k} \rho \psi\right) .
$$

See Theorem 7.10 in [Was82]. The element $\mathscr{L}_{\psi}$ is called the $p$-adic $L$-function attached to $\psi$.

Remark 6.2. Starting from $\mathscr{L}_{\psi}$ above, one may construct a function $L_{p}(s, \psi)$ (which is analytic at all $s \in \mathbb{Z}_{p}$ ) by setting

$$
L_{p}(s, \psi)=\rho_{\mathrm{cyc}}^{1-s}\left(\mathscr{L}_{\psi}\right)
$$

Recall that $L_{n}=L \mathbb{Q}_{n}$ and $L_{\infty}=L \mathbb{Q}_{\infty}$. For a prime $\mathfrak{p}$, let $U_{n, \mathfrak{p}}$ denote the local units inside $\left(L_{n}\right)_{\mathfrak{p}}$. Let $\mathcal{U}_{n}:=\prod_{\mathfrak{p} \mid p} U_{n, \mathfrak{p}}$ be the group of semi-local units and let $\mathcal{V}_{n}=\left(L_{n} \otimes \mathbb{Q}_{p}\right)^{\times}=\prod_{\mathfrak{p} \mid p}\left(L_{n}\right)_{\mathfrak{p}}^{\times}$. By Kummer theory, we have an identification

$$
H^{1}\left(\left(L_{n}\right)_{p}, \mathcal{O}(1)\right) \stackrel{\sim}{\longrightarrow} \widehat{\mathcal{V}}_{n} \text { and } H^{1}\left(\left(\mathbb{Q}_{n}\right)_{p}, T\right) \stackrel{\sim}{\longrightarrow} \mathcal{V}_{n}^{\chi},
$$

where we recall that $\hat{A}$ denotes the $p$-adic completion of an abelian group $A$ and when $A$ is endowed with an action of $\operatorname{Gal}(L / \mathbb{Q})$, we write $A^{\chi}$ for the $\chi$-part of $\hat{A}$. Define $U_{\infty}=\lim _{n} U_{n}$ and $\mathcal{V}_{\infty}=\lim _{n} \mathcal{V}_{n}$, where the inverse limits are taken with respect to the norm maps. The identifications (6.1) above then gives in the limit

$$
H^{1}\left(\mathbb{Q}_{p}, T \otimes \Lambda\right) \stackrel{\sim}{\longrightarrow} \mathcal{V}_{\infty}^{\chi}
$$

Coleman introduced in [Col79] a useful tool which as an input takes a norm coherent sequences in a tower of local fields and gives as an output a power series. More precisely, Coleman defines a $\Lambda$-module homomorphism

$$
\operatorname{cor}_{\infty}^{\psi}: \mathcal{U}_{\infty}^{\psi} \longrightarrow \mathcal{O}[[\Gamma]]
$$

with the property that

$$
\operatorname{cor}_{\infty}^{\psi}\left(\xi_{\infty}^{\psi}\right)=\mathscr{L}_{\psi}
$$


where we recall that $\xi_{\infty}^{\psi} \in \mathcal{U}_{\infty}^{\psi}$ is the norm coherent sequence of cyclotomic units along the tower of fields $\left\{L_{n}\right\}_{n \geq 0}$. Let $\gamma$ be a topological generator of $\Gamma$ as fixed above. If the character $\psi$ is unramified at $p$, then $\operatorname{cor}_{\infty}^{\psi}$ extends uniquely to a homomorphism

$$
\operatorname{cor}_{\infty}^{\psi}: \mathcal{V}_{\infty}^{\psi} \longrightarrow \frac{1}{\gamma-1} \mathcal{O}[[\Gamma]]
$$

See $\S 3$ of [Sol92], $\S 2$ of [Gre92] and $\S 4$ of [Tsu99] for a detailed description of Coleman's map.

We define using (6.5)

$$
\widetilde{\operatorname{col}}_{\infty}^{\psi}=\frac{\gamma-1}{\frac{1}{p} \log _{p}\left(\rho_{\text {cyc }}(\gamma)\right)} \times \operatorname{cor}_{\infty}^{\psi}: \mathcal{V}_{\infty}^{\psi} \rightarrow \Lambda
$$

so that

$$
\widetilde{\operatorname{col}}_{\infty}^{\psi}\left(\xi_{\infty}^{\psi}\right)=\frac{\gamma-1}{\frac{1}{p} \log _{p}\left(\rho_{\mathrm{cyc}}(\gamma)\right)} \times \mathscr{L}_{\psi} \quad \text { and } \quad \widetilde{\operatorname{col}_{\infty}^{\psi}}\left(z_{\infty}^{\psi}\right)=p \mathscr{L}_{\psi}
$$

$z_{\infty}^{\psi} \in \mathcal{V}_{\infty}^{\psi}$ being the collection of wild cyclotomic $p$-units. Note that $\frac{1}{p} \log _{p}\left(\rho_{\text {cyc }}(\gamma)\right) \in$ $\mathbb{Z}_{p}^{\times}$since $\gamma \in \Gamma$ assumed to be a topological generator and since we assumed $p$ is odd.

6.1.2. Elliptic units and Katz's $p$-adic $\boldsymbol{L}$-function. Let $\mathfrak{D}$ be the completion of the ring of integers of the maximal unramified extension of $\mathfrak{F}$ and let $k$ be a quadratic imaginary number field such that $p$ splits in $k / \mathbb{Q}$. Write $p=\wp \wp^{*}$ with $\wp \neq \wp^{*}$. We adapt the notation and hypotheses from $\$ 1.1$, in particular, $k_{\infty}$ is the unique $\mathbb{Z}_{p}$-extension of $k$ which is unramified outside $\wp$ and $\Gamma=\operatorname{Gal}\left(k_{\infty} / k\right)$. Write $k\left(\mathfrak{f} \wp^{\infty}\right)=\bigcup_{n \geq 0} k\left(\mathfrak{f} \wp^{n+1}\right)$ and let

$$
\rho_{E}: \operatorname{Gal}\left(k\left(\mathfrak{f} \wp^{\infty}\right) / k(\mathfrak{f})\right) \longrightarrow \mathbb{Z}_{p}^{\times}
$$

be the character whose construction is sketched in $\S 1.1$; and let $\rho_{\Gamma}$ be its restriction to $\Gamma$. We may similarly define $\rho_{E}^{*}, \Gamma^{*}$ and $\rho_{\Gamma^{*}}$ by replacing $\wp$ by $\wp^{*}$. Set $\mathcal{E}=$ $\operatorname{Gal}\left(k\left(\mathfrak{f} p^{\infty}\right) / k(\mathfrak{f})\right)$ and $\left.\boldsymbol{\Lambda}=\mathfrak{D}[\mathscr{E}]\right]$. We denote the Grössencharacter character attached to the elliptic curve $E$ also by $\rho_{E}$, which should cause no confusion since these two characters are related in a manner described in [Wei56].

For a Grössencharacter $\psi$ of $k$ of type $A_{0}$ (in the sense of [dS87], §II.1) and an integral ideal $\mathfrak{m} \subset k$, the Hecke $L$-series of $\psi$ (with modulus $\mathfrak{m}$ ) is the complex valued function $L_{\infty, \mathfrak{m}}(\psi, s)=\sum \psi(a) \mathbf{N a}{ }^{-s}$, where a runs over all integral ideals relatively prime to $\mathfrak{m}$. Let $d_{k} \in \mathbb{Z}^{-}$be the discriminant of $K$. As before, let $\chi: G_{k} \rightarrow \mathfrak{D}^{\times}$be a Dirichlet character whose order is prime to $p$ and let $\Omega$ be the positive real period of a global minimal model of $E$. For notational simplicity, write $\rho=\rho_{E}$ and $\rho^{*}=\rho_{E}^{*}$.

The following theorem describes the 2 -variable $\wp$-adic $L$-function, first constructed by Katz [Kat76] and Manin and Vishik. 
Theorem 6.3. For $j, k \in \mathbb{Z}$, set $\epsilon=\rho_{E}^{k} \rho_{E}^{* j} \chi$. There is a $\wp$-adic period $\Omega_{\wp} \in \boldsymbol{\Lambda}$ and an element $\mathscr{L}_{\chi} \in \mathbf{\Lambda}$ such that for $0 \leq-j<k$, $\Omega_{\wp}^{j-k} \mathscr{L}_{\chi}\left(\rho^{k} \rho^{* j}\right)=\Omega^{j-k}(k-1) !\left(\frac{\sqrt{-d_{k}}}{2 \pi}\right)^{j} \cdot G(\epsilon)\left(1-\frac{\epsilon(\wp)}{p}\right) \cdot L_{\infty, \wp}\left(\epsilon^{-1}, 0\right)$.

See Theorem II.4.14 in [dS87] for details (e.g., for a definition of $G(\epsilon)$ ) and for the proof.

In this paper, we are only interested in the restriction $\left.\mathscr{L}_{\chi}\right|_{\Gamma}$ of the 2 -variable $p$-adic $L$-function $\mathscr{L}_{\chi}$ to characters of $\Gamma$. Starting from the one-variable $p$-adic $L$-function $\left.\mathscr{L}_{\chi}\right|_{\Gamma}$, we define $\mathfrak{L}_{\wp}(s, \chi)=\left.\mathscr{L}_{\chi}\right|_{\Gamma}\left(\rho_{\Gamma}^{1-s}\right)$.

For $k_{n}$ as in $\S 1.1$, write $L_{n}=L k_{n}$. For a prime $\mathfrak{q}$, let $U_{n, \mathfrak{q}}$ be the local units inside $\left(L_{n}\right)_{\mathfrak{q}}$, and let $U_{n}=\prod_{\mathfrak{q} \mid \wp} U_{n, \mathfrak{q}}$ be the group of semi-local units. Set $u_{\infty}=$ $\lim _{n} U_{n}$. As in $\S 6.1 .1$, we consider Coleman's map

$$
\left.\operatorname{col}_{\infty}^{\chi}: U_{\infty}^{\chi} \otimes_{\mathcal{O}} \mathfrak{D} \longrightarrow \mathfrak{D}[\Gamma]\right]
$$

see $\S$ I.3.5 of [dS87] for a definition of this map. The map $\operatorname{col}_{\infty}^{\chi}$ here is the map " $i$ " of loc.cit. restricted to the $\chi$-parts and to the $\Gamma$-direction.

Let $w_{n} \in L_{n}^{\times}$be the elliptic unit denoted by $\xi_{n}$ by Bley [Ble04], $\S 3$. The collection $\mathfrak{w}_{\infty}^{\chi}:=\left\{\mathfrak{w}_{n}^{\chi}\right\} \in \mathcal{U}_{\infty}^{\chi}$ is called the collection of wild elliptic units along $\Gamma$. As wild cyclotomic units recovers the Kubota-Leopoldt $p$-adic $L$-function, wild elliptic units along $\Gamma$ may be used to obtain the one-variable $p$-adic $L$-function:

$$
\operatorname{cor}_{\infty}^{\chi}\left(\mathfrak{w}_{\infty}^{\chi}\right)=\left.\mathscr{L}_{\chi}\right|_{\Gamma}
$$

This fact has been first proved by Coates and Wiles [CW78]. For the 2-variable version of (6.8), see [Yag82] and [dS87], §IV.

6.2. Height computations for the base field $\mathbb{Q}$ : The case $\chi$ is even. Let $\chi$ be an even Dirichlet character as before. Recall that $\Phi_{n}=\left(\mathbb{Q}_{n}\right)_{p}$, and recall also the fixed place $v_{0}$ of $L$ which is induced from the embedding $\iota_{p}: \mathbb{Q}_{\hookrightarrow} \hookrightarrow \overline{\mathbb{Q}}_{p}$. Write $v_{0}$ for the unique place of $L_{n}$ which lies above $v_{0}$ and define $\mathfrak{R}_{n}=\left(L_{n}\right)_{v_{0}}$. In this section, we construct a particular collection

$$
\Phi=\left\{\phi^{(n)}\right\}_{n} \in H^{1}\left(\mathbb{Q}_{p}, T^{*} \otimes \Lambda\right)={\underset{\lim }{n}}_{H^{1}}\left(\Phi_{n}, T^{*}\right)
$$

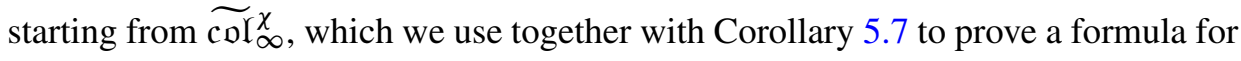
the leading term of an imprimitive Kubota-Leopoldt $p$-adic $L$-function. 
As in (5.1), we have identifications

$$
\begin{aligned}
& H^{1}\left(\Phi_{n}, \mathcal{O}(\chi)\right)=\left(\bigoplus_{v \mid p} H^{1}\left(\left(L_{n}\right)_{v}, \mathcal{O}\right)\right)^{\chi^{-1}} \stackrel{\xi_{\chi}-1}{\longrightarrow} H^{1}\left(\mathfrak{R}_{n}, \mathcal{O}\right)=\operatorname{Hom}\left(G_{\mathfrak{R}_{n}}, \mathcal{O}\right) \\
& =\operatorname{Hom}\left(\widehat{\mathfrak{R}_{n}^{\times}}, \mathcal{O}\right) .
\end{aligned}
$$

Here the direct sum is over the places of $L$ which lie above $p$ with the convention that the unique place of $L_{n}$ above a place $v \mid p$ of $L$ is also denoted by $v$. Also, $\xi_{\chi^{-1}}$ is the projection to the $v_{0}$-coordinate and the final equality is obtained by local class field theory. Furthermore, as in (5.3), we have identifications

$$
\begin{aligned}
H^{1}\left(\Phi_{n}, \mathcal{O}(1) \otimes \chi^{-1}\right) & =\left(\bigoplus_{v \mid p} H^{1}\left(\left(L_{n}\right)_{v}, \mathcal{O}(1)\right)\right)^{\chi} \underset{\xi_{\chi}}{\sim} H^{1}\left(\mathfrak{Q}_{n}, \mathcal{O}(1)\right) \\
& =\widehat{\mathfrak{Q}_{n}^{\times}} \otimes_{\mathbb{Z}_{p}} \mathcal{O},
\end{aligned}
$$

which, put together with the identification above gives isomorphisms

$$
\operatorname{Hom}\left(H^{1}\left(\Phi_{n}, T\right), \mathcal{O}\right) \stackrel{\sim}{\longrightarrow} \operatorname{Hom}\left(\widehat{\mathfrak{R}}_{n}^{\times}, \mathcal{O}\right) \stackrel{\sim}{\sim} H^{1}\left(\Phi_{n}, T^{*}\right)
$$

Note that both isomorphisms in (6.9) depend on the choice of $v_{0}$, yet the compositum of them does not.

Let $\mathcal{U} H^{1}\left(\Phi_{n}, T\right) \subset H^{1}\left(\Phi_{n}, T\right)$ denote submodule of universal norms inside of $H^{1}\left(\Phi_{n}, T\right)$, i.e., the image of the canonical $\Lambda$-module homomorphism

$$
H^{1}\left(\mathbb{Q}_{p}, T \otimes \Lambda\right)=\underset{\leftarrow}{\lim } H^{1}\left(\Phi_{m}, T\right) \longrightarrow H^{1}\left(\Phi_{n}, T\right)
$$

The Coleman map $\widetilde{\operatorname{col}^{\chi}} \underset{\infty}{\lim } H_{m}^{1}\left(\Phi_{m}, T\right)={\underset{\leftarrow}{\lim _{m}}}_{\longleftarrow_{m}} \mathcal{V}_{m}^{\chi} \longrightarrow \Lambda$ induces (since it is $\Lambda$-linear) a $\mathcal{O}\left[\Gamma_{n}\right]$-module homomorphism

$$
\widetilde{\operatorname{col}_{n}^{\chi}}: \mathcal{U} H^{1}\left(\Phi_{n}, T\right) \longrightarrow \mathcal{O}\left[\Gamma_{n}\right]
$$

For a finitely generated $\mathcal{O}\left[\Gamma_{n}\right]$-module $M$, there is a canonical isomorphism

$\mathfrak{b}: \operatorname{Hom}_{\mathcal{O}}(M, \mathcal{O}) \stackrel{\sim}{\longrightarrow} \operatorname{Hom}_{\mathcal{O}\left[\Gamma_{n}\right]}\left(M, \mathcal{O}\left[\Gamma_{n}\right]\right), \quad f \longmapsto\left(m \mapsto \sum_{g \in \Gamma_{n}} f\left(g^{-1} m\right) \cdot g\right)$

(cf. [Bro94], Proposition VI.3.4). Using the isomorphism $\mathfrak{b}$ applied with $M=$ $\mathcal{U} H^{1}\left(\Phi_{n}, T\right)$, we define $\phi^{(n)}$ by requiring $\mathfrak{b}\left(\phi^{(n)}\right)=\widetilde{\mathfrak{c o l l}_{n}^{\chi}}$.

Lemma 6.4. The $\mathcal{O}$-module

$$
H^{1}\left(\Phi_{n}, T\right) / \mathcal{U} H^{1}\left(\Phi_{n}, T\right) \cong \operatorname{coker}\left(H^{1}\left(\mathbb{Q}_{p}, T \otimes \Lambda\right) \rightarrow H^{1}\left(\Phi_{n}, T\right)\right)
$$

is free of rank one. 
Proof. By the long exact sequence of $G_{\mathbb{Q}_{p}}$-cohomology we have $\operatorname{coker}\left(H^{1}\left(\mathbb{Q}_{p}, T \otimes \Lambda\right) \rightarrow H^{1}\left(\Phi_{n}, T\right)\right)=H^{2}\left(\mathbb{Q}_{p}, T \otimes \Lambda\right)\left[\gamma^{p^{n}}-1\right]$.

By Proposition II.1.1 in [Co198] and by local duality, we have

$$
\begin{aligned}
& \left.H^{2}\left(\mathbb{Q}_{p}, T \otimes \Lambda\right)={\underset{\leftarrow}{\leftarrow}}_{\lim } H^{2}\left(\Phi_{n}, T\right)={\underset{\leftarrow}{n}}_{\lim } \operatorname{Hom}\left(H^{0}\left(\Phi_{n}, \mathfrak{F} / \mathcal{O}(\chi)\right), \mathfrak{F} / \mathcal{O}\right)\right) \\
& \left.=\operatorname{Hom}\left(\underset{n}{\lim } H^{0}\left(\Phi_{n}, \mathfrak{F} / \mathcal{O}(\chi)\right), \mathfrak{F} / \mathcal{O}\right)\right) \cong \mathcal{O},
\end{aligned}
$$

which is free of rank one as an $\mathcal{O}$-module.

Remark 6.5. In this remark, we give a further study of the universal norms $\mathfrak{U} H^{1}\left(\Phi_{n}, T\right)$ inside $H^{1}\left(\Phi_{n}, T\right)$. For notational simplicity, we assume $\mathcal{O}=\mathbb{Z}_{p}$; the general case may be treated tensoring all our conclusions in this remark by $\mathcal{O}$. Furthermore, since we assume $\chi(p)=1$ (i.e., $\left.\chi\right|_{G_{\mathbb{Q} p}}=\mathbf{1}$ ), it suffices to study the universal norms $\mathcal{U} H^{1}\left(\Phi_{n}, \mathbb{Z}_{p}(1)\right)$ inside $H^{1}\left(\Phi_{n}, \mathbb{Z}_{p}(1)\right)$.

(i) Let $\varpi_{n} \in \Phi_{n}^{\times}$be a uniformizer which is chosen in a way that $\mathbf{N}_{\Phi_{n} / \Phi_{m}}\left(\varpi_{n}\right)=$ $\varpi_{m}$ for every $n \geq m$. Let $\mathfrak{U}_{\mathfrak{n}}$ the units of $\Phi_{n}$. Kummer theory gives an identification

$$
H^{1}\left(\Phi_{n}, \mathbb{Z}_{p}(1)\right)=\widehat{\Phi_{n}^{\times}}=\varpi_{n}^{\mathbb{Z}_{p}} \times \widehat{\mathcal{U}_{n}} .
$$

Since $p \in H^{1}\left(\mathbb{Q}_{p}, \mathbb{Z}_{p}(1)\right)=p^{\mathbb{Z}_{p}} \times \widehat{\mathbb{Z}}_{p}^{\times}$is a universal norm, it follows from Lemma 6.4 that no local unit (i.e., an element of $\widehat{\mathbb{Z}}_{p}^{\times} \subset \widehat{\mathbb{Q}_{p}^{x}}$ ) besides 1 is a universal norm, and we have $\mathfrak{U} H^{1}\left(\mathbb{Q}_{p}, \mathbb{Z}_{p}(1)\right)=p^{\mathbb{Z}_{p}}$ under the identification above. Set $Y_{0}=\widehat{\mathbb{Z}_{p}^{\times}}$, so that we have a decomposition $H^{1}\left(\mathbb{Q}_{p}, \mathbb{Z}_{p}(1)\right)=$ $\mathfrak{U} H^{1}\left(\Phi_{0}, \mathbb{Z}_{p}(1)\right) \times Y_{0}$ into rank-one $\mathbb{Z}_{p}$-modules. Note that we adopt here the multiplicative notation for these abelian groups.

(ii) For every $n \geq m$, the restriction map

$\operatorname{res}_{\Phi_{m} / \Phi_{n}}: H^{1}\left(\Phi_{m}, \mathbb{Z}_{p}(1)\right) \longrightarrow H^{1}\left(\Phi_{n}, \mathbb{Z}_{p}(1)\right)^{\operatorname{Gal}\left(\Phi_{n} / \Phi_{m}\right)} \hookrightarrow H^{1}\left(\Phi_{n}, \mathbb{Z}_{p}(1)\right)$

is simply the natural injection $\widehat{\Phi_{m}^{\times}} \hookrightarrow \widehat{\Phi_{n}^{\times}}$. When $m=0$, write $\operatorname{res}_{n}$ for $\operatorname{res}_{\Phi_{n} / \mathbb{Q}_{p}}$.

(iii) If $1 \neq u \in \widehat{\mathbb{Z}_{p}^{\times}} \subset H^{1}\left(\mathbb{Q}_{p}, \mathbb{Z}_{p}(1)\right)$, then $\operatorname{res}_{n}(u)$ is not a universal norm. Indeed, if otherwise, $\mathbf{N}_{\Phi_{n} / \mathbb{Q}_{p}}\left(\operatorname{res}_{n}(u)\right)=u^{p^{n}} \in \widehat{\mathbb{Z}_{p}^{\times}}$would then be a universal norm and hence $u^{p^{n}}=1$ by (i). Since $\widehat{\mathbb{Z}}_{p}^{\widehat{x}}$ is torsion-free, it follows that $u=1$. Let

$$
Y_{n}=\operatorname{im}\left(Y_{0} \stackrel{\text { res }_{n}}{\longrightarrow} H^{1}\left(\Phi_{n}, \mathbb{Z}_{p}(1)\right)\right) .
$$


(iv) The quotient $H^{1}\left(\Phi_{n}, \mathbb{Z}_{p}(1)\right) / Y_{n}=\widehat{\Phi_{n}^{\times}} / \operatorname{im}\left(\widehat{\mathbb{Z}}_{p}^{\times} \hookrightarrow \widehat{\Phi_{n}^{\times}}\right)$is torsion-free. Indeed, if an element of the quotient $\widehat{\Phi_{n}^{\times}} / \operatorname{im}\left(\widehat{\mathbb{Z}_{p}^{\times}} \hookrightarrow \widehat{\Phi_{n}^{\times}}\right)$represented by $x \in$ $\widehat{\Phi_{n}^{\times}}-\widehat{\mathbb{Z}}_{p}^{\times}$is $p$-torsion, so that $x^{p} \in \widehat{\mathbb{Z}}_{p}^{\times}$, then we would have $\mu_{p} \subset \Phi_{n}^{\times}$, which is not true. Hence, $Y_{n}$ is a free rank-one direct summand of $H^{1}\left(\Phi_{n}, \mathbb{Z}_{p}(1)\right)$.

(v) By Lemma 6.4, we have

$$
\operatorname{rank}_{\mathbb{Z}_{p}} \mathfrak{U} H^{1}\left(\Phi_{n}, \mathbb{Z}_{p}(1)\right)=\operatorname{rank}_{\mathbb{Z}_{p}} H^{1}\left(\Phi_{n}, \mathbb{Z}_{p}(1)\right)-1 .
$$

Using (iii), (iv) and (6.10), we conclude that

$$
H^{1}\left(\Phi_{n}, \mathbb{Z}_{p}(1)\right)=\mathfrak{U} H^{1}\left(\Phi_{n}, \mathbb{Z}_{p}(1)\right) \times Y_{n}
$$

as $\mathbb{Z}_{p}$-modules.

Remark $6.5(\mathrm{v})$ ensures that one may extend $\phi^{(n)}: \mathfrak{U} H^{1}\left(\Phi_{n}, T\right) \rightarrow \mathcal{O}$ to a homomorphism $H^{1}\left(\Phi_{n}, T\right) \rightarrow \mathcal{O}$, by declaring $\phi^{(n)}(c)=0$ for $c \in Y_{n}$. Note in particular for $n=0$ that the map $\phi^{(0)}=\widetilde{\operatorname{col}_{0}^{\chi}}: H^{1}\left(\mathbb{Q}_{p}, T\right) \stackrel{\sim}{\longrightarrow} \widehat{L_{v_{0}}^{x}} \otimes_{\mathbb{Z}_{p}} \mathcal{O} \rightarrow \mathcal{O}$ (which is extended from $\mathcal{U} H^{1}\left(\mathbb{Q}_{p}, T\right)$ as described above) is unramified since it is identically zero on the units $\widehat{\mathcal{O}_{L_{v_{0}}}^{\times}} \otimes_{\mathbb{Z}_{p}} \mathcal{O}$ by construction (as explained in Remark 6.5(i)).

Let $\varpi_{v_{0}} \in L_{v_{0}}^{\times}$be a uniformizer and set $\alpha\left(v_{0}\right)=\widetilde{\operatorname{cor}_{0}^{\chi}}\left(\varpi_{v_{0}}\right) \in \mathcal{O}$. Note that the value $\widetilde{\operatorname{col}_{0}^{x}}\left(\varpi_{v_{0}}\right)$ is well defined thanks to the discussion in the preceding paragraph. Let $\operatorname{cor}_{0}^{\chi} \in \widetilde{H}_{f}^{1}\left(\mathbb{Q}, T^{*}\right)$ be the element which maps to $\alpha\left(v_{0}\right)$ under the compositum of the isomorphisms (4.2). Furthermore, one may verify without difficulty that the collection $\Phi=\left\{\phi^{(n)}\right\}$ chosen as in this section is norm-coherent and the Rubin-style formula we proved (Corollary 5.7) applies with the particular $\Phi$ we have constructed. Before stating the theorem we prove using these facts, we first define what we call the "imprimitive $p$-adic $L$-function".

Definition 6.6. For $\mathscr{L}_{\chi} \in \Lambda$ as above and for any topological generator $\gamma \in \Gamma$, write $\widetilde{\mathscr{L}}_{\chi}:=\frac{\gamma-1}{\frac{1}{p} \log _{p} \rho_{\mathrm{cyc}}(\gamma)} \times \mathscr{L}_{\chi} \in \Lambda$, and define the imprimitive $p$-adic L-function to be

$$
\widetilde{L}_{p}(s, \chi)=\rho_{\text {cyc }}^{1-s}\left(\widetilde{\mathscr{L}}_{\chi}\right) .
$$

Note that,

- $\widetilde{L}_{p}(s, \chi)$ is an Iwasawa function,

- $\left.\frac{d}{d s} \widetilde{L}_{p}(s, \chi)\right|_{s=1}$ does not depend on the choice of $\gamma$.

Theorem 6.7. Suppose $\chi(p)=1$ and let $\tilde{L}_{p}(s, \chi)$ be the imprimitive $p$-adic $L$ function defined as above. Then

$$
\tilde{L}_{p}^{\prime}(1, \chi)=\left\langle c_{1}^{\chi}, \operatorname{cor}_{0}^{\chi}\right\rangle_{\mathrm{Nek}} .
$$


Proof. As in $\S 5$, let $\mu_{\xi, \Phi}$ be the measure on $\Gamma$ attached to $\xi=\xi_{\infty}^{\chi}$ and $\Phi$ we chose as above, let $\Re_{\xi, \Phi} \in \Lambda$ be the associated power series and let $L_{\xi, \Phi}(\eta)$ denote the ' $p$-adic $L$-function' on the characters $\eta: \Gamma \rightarrow \mathbb{Z}_{p}^{\times}$. We then have

$$
\begin{aligned}
& \mathfrak{P}_{\xi, \Phi}=\widetilde{\operatorname{col}}_{\infty}^{\chi}\left(\xi_{\infty}^{\chi}\right)=\frac{\gamma-1}{\frac{1}{p} \log _{p} \rho_{\mathrm{cyc}}(\gamma)} \times \operatorname{col}_{\infty}^{\chi}\left(\xi_{\infty}^{\chi}\right) \\
& =\frac{\gamma-1}{\frac{1}{p} \log _{p} \rho_{\text {cyc }}(\gamma)} \times \mathscr{L}_{\chi} .
\end{aligned}
$$

We therefore see that

$$
\left.\frac{d}{d s} \rho_{\text {cyc }}^{s}\left(\Re_{\xi, \Phi}\right)\right|_{s=0}=p \cdot \mathbf{1}\left(\mathscr{L}_{\chi}\right)=p \cdot L_{p}(1, \chi)=\left.\frac{d}{d s} \widetilde{L}_{p}(s, \chi)\right|_{s=1},
$$

where we have the first equality because $\frac{d}{d s} \rho_{\text {cyc }}^{s}=\log _{p} \rho_{\text {cyc }} \cdot \rho_{\text {cyc }}^{s}$, the second thanks to our definition of $L_{p}(s, \chi)$ (see Remark 6.2).

On the other hand, we have $\left.\frac{d}{d s} \rho_{\text {cyc }}^{s}\left(\Re_{\xi, \Phi}\right)\right|_{s=0}=L_{\xi, \Phi}^{\prime}(\mathbf{1})$ by Remark 5.4, and the theorem follows combining (6.11) and Corollary 5.7.

Remark 6.8. When $\chi$ is an even character with $\chi(p)=1$, the exceptionality that Nekovár's extended Selmer groups detect are not due to an honest exceptional zero of the associated Kubota-Leopoldt $p$-adic $L$-function, but rather due to the fact that the extended Selmer groups correspond to an imprimitive $p$-adic $L$-function.

6.3. Height computations for the base field $\mathbb{Q}$ : The case $\chi$ is odd. We suppose now that $\chi: G_{\mathbb{Q}} \rightarrow \mathcal{O}^{\times}$is an odd Dirichlet character whose order is prime to $p$ and which has the property that $\chi(p)=1$. Keeping the notation of $\$ 2.1$ and $\$ 2.2$, we have the following identifications as in Proposition 2.10 and Corollary 2.11:

$$
\begin{gathered}
\widetilde{H}_{f}^{1}(\mathbb{Q}, T)=H_{\mathcal{F}_{\text {can }}}^{1}(\mathbb{Q}, T)=\left(\mathcal{O}_{L}[1 / p]^{\times}\right)^{\chi}, \\
H^{0}\left(\mathbb{Q}_{p}, \mathcal{O}(\chi)\right) \stackrel{\sim}{\longrightarrow} \widetilde{H}_{f}^{1}\left(\mathbb{Q}, T^{*}\right) .
\end{gathered}
$$

In particular, $\widetilde{H}_{f}^{1}\left(\mathbb{Q}, T^{*}\right)$ is a free $\mathcal{O}$-module of rank one. Also, since $\chi$ is odd and $\chi(p)=1$, the $\mathcal{O}$-module $\tilde{H}_{f}^{1}(\mathbb{Q}, T)$ is also free of rank one.

The assumption that $\chi(p)=1$ implies that the prime $p$ splits completely in $L / \mathbb{Q}$. Let $\wp \subset L$ be any prime above $p$ and let $\iota_{\wp}: L \hookrightarrow L_{\wp}=\mathbb{Q}_{p}$ be the induced embedding. Let $h$ denote the class number of $L$, and let $x \in \mathcal{O}_{L}[1 / p]^{\times}$be such that $\mathcal{O}_{L} \cdot x=\wp^{h}$. Define

$$
z=e_{\chi} \cdot x \in\left(\mathcal{O}_{L}[1 / p]^{\times}\right)^{\chi}=\widetilde{H}_{f}^{1}(\mathbb{Q}, T) \quad \text { and } \quad z_{0}=\frac{1}{h} \cdot z \in \widetilde{H}_{f}^{1}(\mathbb{Q}, T) \otimes \mathbb{Q}_{p} .
$$


It is not hard to see that the $\mathscr{L}$-invariant (cf. [Gre94], §1)

$$
\mathscr{L}:=\frac{\log _{p}\left(\iota_{\wp}(z)\right)}{\operatorname{ord}_{\wp}(z)}=\log _{p}\left(\iota_{\wp}\left(z_{0}\right)\right) \in \mathfrak{F}=\operatorname{Frac}(\mathcal{O})
$$

is independent of the choice of the place $\wp$ and the choice of $x$.

Let $f=f_{L}$ be the conductor of the abelian field $L$. We regard the character $\chi$ as a character of the group $\Delta_{f}:=\operatorname{Gal}\left(\mathbb{Q}\left(\mu_{f}\right) / \mathbb{Q}\right)$ via

$$
\chi: \Delta_{f} \longrightarrow \operatorname{Gal}(L / \mathbb{Q}) \rightarrow \mathcal{O}^{\times}
$$

and define the tame Stickelberger element

$$
\theta_{f}=\sum_{a \in(\mathbb{Z} / f \mathbb{Z})^{\times} \cong \Delta_{f}}\left(\frac{\langle a\rangle}{f}-\frac{1}{2}\right) \delta_{a}^{-1} \in \mathcal{O}\left[\Delta_{f}\right],
$$

so that

$$
\chi\left(\theta_{f}\right)=B_{1, \chi^{-1}}=-L\left(0, \chi^{-1}\right),
$$

where $B_{1, \chi^{-1}}$ is the generalized Bernoulli number.

Fixing generators $g_{\chi}$ of $\mathcal{O}(\chi)$ and $g_{\chi^{-1}}$ of $\mathcal{O}\left(\chi^{-1}\right)$, and using the fact that $\chi(p)=$ 1 , we obtain isomorphisms

$$
g_{\chi}: H^{i}\left(\mathbb{Q}_{p}, T\right) \stackrel{\sim}{\longrightarrow} H^{i}\left(\mathbb{Q}_{p}, \mathcal{O}(1)\right) \quad \text { and } \quad g_{\chi^{-1}}: H^{i}\left(\mathbb{Q}_{p}, T^{*}\right) \stackrel{\sim}{\longrightarrow} H^{i}\left(\mathbb{Q}_{p}, \mathcal{O}\right)
$$

for every $i \geq 0$. We choose $g_{\chi}$ and $g_{\chi^{-1}}$ so that the following diagram is commutative:

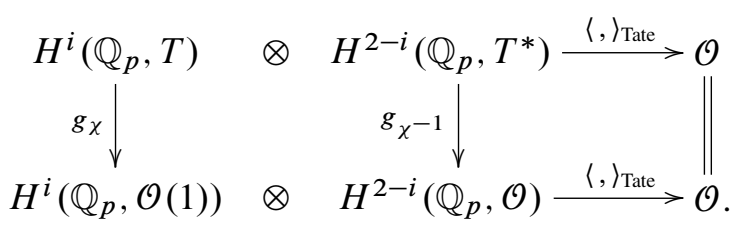

Via the identifications above, we view $\chi\left(\theta_{f}\right)$ as an element of $\tilde{H}_{f}^{1}\left(\mathbb{Q}, T^{*}\right)$.

Let $\langle,\rangle_{\text {Nek }}$ be Nekovář's height pairing as in $\$ 2.1 .3$ above. We write $\langle,\rangle_{\text {Nek }}$ also for the induced pairing

$$
\left(\tilde{H}_{f}^{1}(\mathbb{Q}, T) \otimes \mathfrak{F}\right) \otimes\left(\tilde{H}_{f}^{1}\left(\mathbb{Q}, T^{*}\right) \otimes \mathfrak{F}\right) \stackrel{\langle,\rangle_{\mathrm{Nek}}}{\longrightarrow} \mathfrak{F} .
$$

Theorem 6.9. $\left\langle z_{0}, \chi\left(\theta_{f}\right)\right\rangle_{\mathrm{Nek}}=-\mathscr{L} \cdot L\left(0, \chi^{-1}\right)$.

Proof. The statement of this theorem is equivalent to the assertion that

$$
\left\langle z, \chi\left(\theta_{f}\right)\right\rangle_{\mathrm{Nek}}=\log _{p}\left(\iota_{\wp}(z)\right) \cdot \chi\left(\theta_{f}\right) .
$$


As we have recalled in $\S 2.1 .3$, we have $\left\langle z_{0}, \chi\left(\theta_{f}\right)\right\rangle_{\mathrm{Nek}}=\left\langle\beta^{1}\left(z_{0}\right), \chi\left(\theta_{f}\right)\right\rangle_{\mathrm{PT}}$, where

$$
\beta^{1}: \widetilde{H}_{f}^{1}(\mathbb{Q}, T) \longrightarrow \widetilde{H}_{f}^{2}(\mathbb{Q}, T) \otimes \Gamma
$$

is the Bockstein map which is defined as follows:

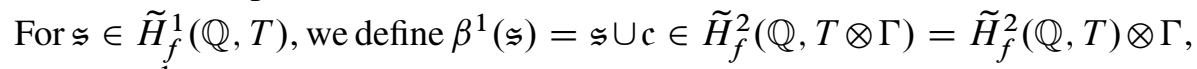
where $c \in H^{1}(\mathbb{Q}, \Gamma)=\operatorname{Hom}\left(G_{\mathbb{Q}}, \Gamma\right)$ is the tautological homomorphism $c: G_{\mathbb{Q}} \rightarrow$ $\Gamma$. One similarly defines

$$
\beta_{p}^{1}: H^{1}\left(\mathbb{Q}_{p}, T\right) \longrightarrow H^{2}\left(\mathbb{Q}_{p}, T\right) \otimes \Gamma
$$

by taking cup product with the element $c_{p} \in H^{1}\left(\mathbb{Q}_{p}, \Gamma\right)=\operatorname{Hom}\left(G_{\mathbb{Q}_{p}}, \Gamma\right)$, which is the restriction of $c$ to $G_{\mathbb{Q}_{p}}$. We then have the following commutative diagram:

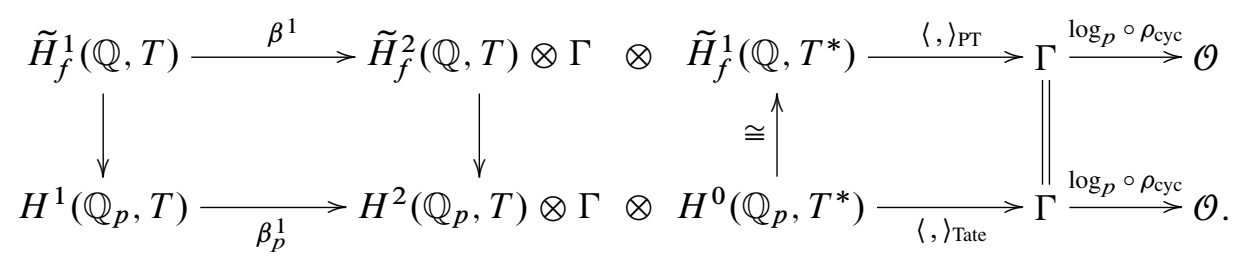

Here, the square on the left is commutative thanks to the description of $\beta^{1}$ and $\beta_{p}^{1}$ above, and the square on the right is commutative by the definition of the Poitou-Tate global pairing as the sum of local invariants, and thanks to the fact that $H^{2}\left(\mathbb{Q}_{\ell}, T\right)=0$ for $\ell \mid \mathfrak{f}_{\chi}$. The proof of the theorem follows from the following lemma, whose first part is a restatement of [Nek06], 11.3.5.3, and second part is Lemma II.1.4.5 in [Kat93]:

Lemma 6.10. Suppose $\alpha \in H^{1}\left(\mathbb{Q}_{p}, \mathcal{O}(1)\right)=\widehat{\mathbb{Q}_{p}^{x}}$, and suppose $a_{p}: \widehat{\mathbb{Q}_{p}^{x}} \rightarrow G_{\mathbb{Q}_{p}}^{\mathrm{ab}}$ is the local reciprocity map as before.

(i) $\operatorname{inv}_{p}\left(\beta_{p}^{1}(\alpha)\right)=\operatorname{inv}_{p}\left(\alpha \cup \mathfrak{c}_{p}\right)=\mathfrak{c}_{p}\left(\mathfrak{a}_{p}(\alpha)\right)$.

(ii) $\log _{p} \circ \rho_{\mathrm{cyc}} \circ c_{p}\left(\mathfrak{a}_{p}(\alpha)\right)=\log _{p}(\alpha)$.

Remark 6.11. The interpolation property that the $p$-adic $L$-function $L_{p}\left(s, \chi^{-1} \omega\right)$ satisfies (see Definition 6.1), along with our assumption that $\chi(p)=1$ forces the Kubota-Leopoldt $p$-adic $L$-function $L_{p}\left(0, \chi^{-1} \omega\right)$ to vanish at $s=0$. The theorem of Ferrero-Greenberg [FG78] combined with a result of Gross and Koblitz [GK79] shows that

$$
\left.\frac{d}{d s} L_{p}\left(s, \chi^{-1} \omega\right)\right|_{s=0}=-\mathscr{L} \cdot L\left(0, \chi^{-1}\right) .
$$

Thus, Theorem 6.9 implies that

$$
\left.\frac{d}{d s} L_{p}\left(s, \chi^{-1} \omega\right)\right|_{s=0}=\left\langle z_{0}, \chi\left(\theta_{f}\right)\right\rangle_{\mathrm{Nek}}
$$


This provides us with a new interpretation of the Ferrero-Greenberg theorem. Of course, it would be desirable to prove first a Rubin-style formula (as we did in §5) in this setting and from that deduce (6.15) and the Ferrero-Greenberg theorem (as we prove a $p$-adic Kronecker formula from a Rubin-style formula in $\$ 6.2$ above and $\S 6.4$ below).

Remark 6.12. Suppose in this remark that our base field $K$ is an arbitrary totally real number field and $\chi: G_{K} \rightarrow \mathcal{O}^{\times}$is a totally odd character which has finite prime-to- $p$ order. Assume further that $\chi(\wp)=1$ for exactly one prime $\wp \subset K$ above $p$. In this setting, Gross conjectured in [Gro81] a formula for the leading coefficient $L_{p}^{\prime}\left(0, \chi^{-1} \omega\right)$ of the Deligne-Ribet $p$-adic $L$-function $L_{p}\left(s, \chi^{-1} \omega\right)$ at $s=0$, and Darmon, Dasgupta and Pollack recently announced a proof of a portion of this conjecture. Using their result, we may express $L_{p}^{\prime}\left(0, \chi^{-1} \omega\right)$ in terms of Nekovář's heights exactly as we did above for the Kubota-Leopoldt $p$-adic $L$-function when $K=\mathbb{Q}$.

On the other hand, if one succeeds in proving a Rubin-style formula in this setting ${ }^{4}$, then one in turn would obtain an alternative proof of Gross' conjecture.

6.4. Height computations for a totally imaginary base field $\boldsymbol{k}$. We keep the notation from $\S 6.1 .2$. Every Dirichlet character $\chi$ of $G_{k}$ behaves like an even character and the results we presented in $\S 5$ and $\$ 6.2$ extend to this case without an extra effort. Replacing the cyclotomic units by elliptic units, and the results of [BG03] by that of [Ble06]; the results of [Sol92] by that of [Ble04], one may prove the following formula:

Theorem 6.13. Suppose $\chi(\wp)=1$. Then

$$
\tilde{\mathfrak{R}}_{p}^{\prime}(1, \chi)=\left\langle e_{1}^{\chi}, \operatorname{cor}_{0}^{\chi}\right\rangle_{\mathrm{Nek}} .
$$

Here we follow the notation from $§ 6.1 .2$. Namely,

- $e_{1}$ is the (tame) elliptic unit which is denoted by $\mathbf{N}_{k(\mathfrak{f}) / L} \psi(1, \mathfrak{f}, \mathfrak{a})$ in [Ble04] and $e_{1}^{\chi} \in \tilde{H}_{f}^{1}(k, T)=H_{\widetilde{F}_{\text {can }}}^{1}(k, T)=L^{\times, \chi}$ is the $\chi$ part of $e_{1}$,

- $\operatorname{cor}_{0}^{\chi} \in \tilde{H}_{f}^{1}\left(k, T^{*}\right)$ is the element which is obtained from the Coleman map (as in $\S 6.2$ ),

- $\widetilde{\mathfrak{R}}_{p}(s, \chi)=\frac{\rho_{\mathrm{cyc}}^{1-s}(\gamma)-1}{\frac{1}{p} \log _{p} \rho_{\mathrm{cyc}}(\gamma)} \cdot \mathfrak{L}_{p}(s, \chi)$ is the imprimitive (one-variable) Katz $p$ adic $L$-function, where $\mathfrak{R}_{p}(s, \chi)$ is the restriction of the two-variable $p$-adic $L$-function to $\Gamma$.

\footnotetext{
${ }^{4}$ It is expected that obtaining a Rubin-style formula for a general totally real $k$ (and for a totally odd character $\chi$ ) should not be any harder than proving such a formula for $k=\mathbb{Q}$.
} 
Remark 6.14. Suppose $E_{/ \mathbb{Q}}$ is an elliptic curve and only in this remark, let $T=T_{p}(E)$ be the $p$-adic Tate-module of $E$. Let $L_{p}(E, s)$ denote the Mazur-Tate-Teitelbaum $p$-adic $L$-function attached to $E$. Assume that $E$ has split multiplicative reduction at $p$. In this case, $L_{p}(E, s)$ has an exceptional zero at $s=1$ which is forced by the interpolation property. The Mazur-Tate-Teitelbaum conjecture (now a theorem of Greenberg and Stevens [GS93]) asserts that

$$
\left.\frac{d}{d s} L_{p}(E, s)\right|_{s=1}=\mathscr{L}_{E} \cdot \frac{L(E, 1)}{\Omega_{E}^{+}}
$$

where $\mathscr{L}_{E}$ is the $\mathscr{L}$-invariant, $L(E, 1)$ is the value of the Hasse-Weil $L$-function at $s=1$ and $\Omega_{E}^{+}$is the real period of $E$. Let

$$
\langle,\rangle_{\text {Tate }}: H^{1}\left(\mathbb{Q}_{p}, T\right) \otimes H^{1}\left(\mathbb{Q}_{p}, T^{*}\right) \longrightarrow \mathbb{Z}_{p}
$$

denote Tate's local cup-product pairing. M. Kurihara has kindly explained us how one may interpret the quantity on the right in (6.16) as the local Tate pairing calculated on Kato's zeta-element $\mathcal{Z}_{0} \in H^{1}\left(\mathbb{Q}_{p}, T\right)$ and another special element $\alpha \in H^{1}\left(\mathbb{Q}_{p}, T^{*}\right)$ (which we do not define here). Using this observation, Kurihara was able to give another proof of the Mazur-Tate-Teitelbaum conjecture (6.16).

If one succeeds in proving a Rubin-style formula in this setting, one could globalize Kurihara's calculation with Kato's zeta-element $\mathcal{Z}_{0}$ and the element $\alpha$, so as to obtain a $p$-adic Gross-Zagier formula in the presence of an exceptional zero, i.e., relate Nekovarr's height pairing to the Mazur-Tate-Teitelbaum $p$-adic $L$-function via

(1) a Rubin-style formula to relate heights to local Tate pairing,

(2) then using Kurihara's local calculation,

much in the spirit of [BD96], [BD97].

\section{References}

[BD96] M. Bertolini and H. Darmon, Heegner points on Mumford-Tate curves. Invent. Math. 126 (1996), no. 3, 413-456. Zbl 0882.11034 MR 1419003

[BD97] M. Bertolini and H. Darmon, A rigid analytic Gross-Zagier formula and arithmetic applications. With an appendix by Bas Edixhoven. Ann. of Math. (2) 146 (1997), no. 1, 111-147. Zbl 1029.11027 MR 1469318

[Ble04] W. Bley, Wild Euler systems of elliptic units and the equivariant Tamagawa number conjecture. J. Reine Angew. Math. 577 (2004), no. 2, 117-146. Zbl 1056.11035 MR 2108215

[Ble06] W. Bley, Equivariant Tamagawa number conjecture for abelian extensions of a quadratic imaginary field. Doc. Math. 11 (2006), 73-118. Zbl 1178.11070 MR 2226270 
[Bro94] K. S. Brown, Cohomology of groups. Corrected reprint of the 1982 original, Grad. Texts in Math. 87, Springer-Verlag, New York 1994. Zbl 0584.20036 MR 1324339

[BG03] D. Burns and C. Greither, On the equivariant Tamagawa number conjecture for Tate motives. Invent. Math. 153 (2003), no. 2, 303-359. Zbl 1142.11076 MR 1992015

[CW78] J. Coates and A. Wiles, On $p$-adic L-functions and elliptic units. J. Austral. Math. Soc. Ser. A 26 (1978), no. 1, 1-25. Zbl 0442.12007 MR 0510581

[Col79] R. F. Coleman, Division values in local fields. Invent. Math. 53 (1979), no. 2, 91-116. Zbl 0429.12010 MR 0560409

[Co198] P. Colmez, Théorie d'Iwasawa des représentations de de Rham d'un corps local. Ann. of Math. (2) 148 (1998), no. 2, 485-571. Zbl 0928.11045 MR 1668555

[dS87] E. de Shalit, Iwasawa theory of elliptic curves with complex multiplication. p-adic $L$ functions. Perspect. Math. 3, Academic Press, Boston, Mass., 1987. Zbl 0674.12004 MR 0917944

[FG78] B. Ferrero and R. Greenberg, On the behavior of p-adic L-functions at s=0. Invent. Math. 50 (1978), 91-102. Zbl 0441.12003 MR 0516606

[Gre89] R. Greenberg, Iwasawa theory for $p$-adic representations. In Algebraic number theory, Adv. Stud. Pure Math. 17, Academic Press, Boston, Mass., 1989, 97-137. Zbl 0739.11045 MR 1097613

[Gre94] R. Greenberg, Trivial zeros of $p$-adic $L$-functions. In $p$-adic monodromy and the Birch and Swinnerton-Dyer conjecture (Boston 1991), Contemp. Math. 165, Amer. Math. Soc., Providence, R.I., 1994, 149-174. Zbl 0838.11070 MR 1279608

[GS93] R. Greenberg and Glenn Stevens, $p$-adic $L$-functions and $p$-adic periods of modular forms. Invent. Math. 111 (1993), no. 2, 407-447. Zbl 0778.11034 MR 1198816

[Gre92] C. Greither, Class groups of abelian fields, and the main conjecture. Ann. Inst. Fourier (Grenoble) 42 (1992), no. 3, 449-499. Zbl 0729.11053 MR 1182638

[Gro81] B. H. Gross, $p$-adic $L$-series at $s=0$. J. Fac. Sci. Univ. Tokyo Sect. IA Math. 28 (1982), no. 2, 979-994. Zbl 0507.12010 MR 0656068

[GK79] B. H. Gross and N. Koblitz, Gauss sums and the $p$-adic $\Gamma$-function. Ann. of Math. (2) 109 (1979), no. 3, 569-581. Zbl 0406.12010 MR 0534763

[GZ86] B. H. Gross and D. B. Zagier, Heegner points and derivatives of $L$-series. Invent. Math. 84 (1986), no. 2, 225-320. Zbl 0608.14019 MR 0833192

[How04] B. Howard, Derived $p$-adic heights and $p$-adic $L$-functions. Amer. J. Math. 126 (2004), no. 6, 1315-1340. Zbl 1073.11037 MR 2102397

[How05] B. Howard, The Iwasawa theoretic Gross-Zagier theorem. Compos. Math. 141 (2005), no. 4, 811-846. Zbl 02211027 MR 2148200

[Kat93] K. Kato, Lectures on the approach to Iwasawa theory for Hasse-Weil $L$-functions via $B_{\mathrm{dR}}$. I. In Arithmetic algebraic geometry (Trento, 1991), Lecture Notes in Math. 1553, Springer-Verlag, Berlin 1993, 50-163. Zbl 0815.11051 MR 1338860

[Kat04] K. Kato, $p$-adic Hodge theory and values of zeta functions of modular forms. Cohomologies p-adiques et applications arithmétiques. III, Astérisque 295, Soc. Math. France, Paris 2004, 117-290. Zbl 1142.11336 MR 2104361

[Kat76] N. M. Katz, p-adic interpolation of real analytic Eisenstein series. Ann. of Math. (2) 104 (1976), no. 5, 459-571. Zbl 0354.14007 MR 0506271 
[Mat89] H. Matsumura, Commutative ring theory. Translated from the Japanese by M. Reid, Cambridge Stud. Adv. Math. 8, Cambridge University Press, Cambridge 1989. Zbl 0666.13002 MR 1011461

[MR04] B. Mazur and K. Rubin, Kolyvagin systems. Mem. Amer. Math. Soc. 168 (2004), no. 799. Zbl 1055.11041 MR 2031496

[Nek93] J. Nekováŕ, On p-adic height pairings. In Séminaire de théorie des nombres, Paris, 1990-91, Progr. Math. 108, Birkhäuser, Boston, Mass., 1993, 127-202. Zbl 0859.11038 MR 1263527

[Nek95] J. Nekovář, On the p-adic height of Heegner cycles. Math. Ann. 302 (1995), no. 4, 609-686. Zbl 0841.11025 MR 1343644

[Nek06] J. Nekovář, Selmer complexes. Astérisque 310 (2006). Zbl 05161833 MR 2333680

[PR87] B. Perrin-Riou, Points de Heegner et dérivées de fonctions $L$ p-adiques. Invent. Math. 89 (1987), no. 3, 455-510. Zbl 0645.14010 MR 0903381

[PR92] B. Perrin-Riou, Théorie d'Iwasawa et hauteurs p-adiques. Invent. Math. 109 (1992), no. 1, 137-185. Zbl 0781.14013 MR 1168369

[PR93] B. Perrin-Riou, Fonctions $L$ p-adiques d'une courbe elliptique et points rationnels. Ann. Inst. Fourier (Grenoble) 43 (1993), no. 4, 945-995. Zbl 0840.11024 MR 1252935

[PR94] B. Perrin-Riou, Théorie d'Iwasawa des représentations $p$-adiques sur un corps local. With an appendix by Jean-Marc Fontaine. Invent. Math. 115 ( 1994), no. 1, 81-161, 1994. Zbl 0838.11071 MR 1248080

[PR83] B. Perrin-Riou, Descente infinie et hauteur $p$-adique sur les courbes elliptiques à multiplication complexe. Invent. Math. 70 (1982/83), no. 3, 369-398. Zbl 0547.14025 MR 0683689

[Rub92] K. Rubin, $p$-adic $L$-functions and rational points on elliptic curves with complex multiplication. Invent. Math. 107 (1992), no. 2, 323-350. Zbl 0770.11033 MR 1144427

[Rub94] K. Rubin, Abelian varieties, p-adic heights and derivatives. In Algebra and number theory (Essen, 1992), Walter de Gruyter, Berlin 1994, 247-266. Zbl 0829.11034 MR 1285370

[Rub00] K. Rubin, Euler systems. Hermann Weyl Lectures, The Institute for Advanced Study, Ann. of Math. Stud. 147, Princeton University Press, Princeton, N.J., 2000. Zbl 0977.11001 MR 1749177

[Sol92] D. Solomon, On a construction of $p$-units in abelian fields. Invent. Math. 109 (1992), no. 2, 329-350. Zbl 0772.11043 MR 1172694

[So194] D. Solomon, Galois relations for cyclotomic numbers and $p$-units. J. Number Theory 46 (1994), no. 2, 158-178. Zbl 0807.11054 MR 1269250

[Tsu99] T. Tsuji, Semi-local units modulo cyclotomic units. J. Number Theory 78 (1999), no. 1, 1-26. Zbl 0948.11042 MR 1706941

[Was82] L. C. Washington, Introduction to cyclotomic fields. Grad. Texts in Math. 83, SpringerVerlag, New York 1982. Zbl 0484.12001 MR 0718674

[Wei56] A. Weil, On a certain type of characters of the idèle-class group of an algebraic numberfield. In Proceedings of the international symposium on algebraic number theory, Tokyo \& Nikko, 1955; Science Council of Japan, Tokyo 1956, 1-7. Zbl 0073.26303 MR 0083523 
Vol. 87 (2012)

[Yag82] R. I. Yager, On two variable $p$-adic $L$-functions. Ann. of Math. (2) 115 (1982), no. 2, 411-449. Zbl 0496.12010 MR 0647813

Received May 22, 2009

Kâzim Büyükboduk, Koc University, Mathematics, Rumeli Feneri Yolu, 34450, Sariyer, Istanbul, Turkey

E-mail: kbuyukboduk@ku.edu.tr

http://home.ku.edu.tr/ kbuyukboduk/Site/welcome.html 